\title{
AN ASSMUS-MATTSON THEOREM FOR CODES OVER COMMUTATIVE ASSOCIATION SCHEMES
}

\author{
JOHN VINCENT S. MORALES AND HAJIME TANAKA
}

\begin{abstract}
We prove an Assmus-Mattson-type theorem for block codes where the alphabet is the vertex set of a commutative association scheme (say, with $s$ classes). This in particular generalizes the Assmus-Mattson-type theorems for $\mathbb{Z}_{4}$-linear codes due to Tanabe (2003) and Shin, Kumar, and Helleseth (2004), as well as the original theorem by Assmus and Mattson (1969). The weights of a code are $s$-tuples of non-negative integers in this case, and the conditions in our theorem for obtaining $t$-designs from the code involve concepts from polynomial interpolation in $s$ variables. The Terwilliger algebra is the main tool to establish our results.
\end{abstract}

\section{INTRODUCTION}

We begin by recalling the famous Assmus-Mattson theorem which relates linear codes and combinatorial designs:

Theorem 1.1 (Assmus and Mattson [1, Theorem 4.2]). Let $C$ be a linear code of length $n$ over $\mathbb{F}_{q}$ with minimum weight $\delta$. Let $C^{\perp}$ denote the dual code of $C$, with minimum weight $\delta^{*}$. Suppose that an integer $t(1 \leqslant t \leqslant n)$ is such that there are at most $\delta-t$ weights of $C^{\perp}$ in $\{1,2, \ldots, n-t\}$, or such that there are at most $\delta^{*}-t$ weights of $C$ in $\{1,2, \ldots, n-t\}$. Then the supports of the words of any fixed weight in $C$ form a t-design (with possibly repeated blocks).

We remark that [1, Theorem 4.2] also includes a criterion for obtaining simple $t$ designs, but we will not pay much attention in this paper to the simplicity of the resulting designs. There are several proofs and strengthenings of Theorem 1.1 see, e.g., [11, 10, 31, 2, 35, 23, 37]. The purpose of this paper is to establish a theorem which unifies many of the known generalizations and extensions of Theorem 1.1

Constructing $t$-designs from codes received renewed interest when Gulliver and Harada [17] and Harada [18 found new 5-designs by computer from the lifted Golay code of length 24 over $\mathbb{Z}_{4}$ (among others). Their constructions were later explained and generalized further by Bonnecaze, Rains, and Solé [6]. Motivated by these results, Tanabe 34] obtained an Assmus-Mattson-type theorem for $\mathbb{Z}_{4}$-linear codes with respect to the symmetrized weight enumerator. Tanabe's theorem can indeed capture the 5 -designs from the lifted Golay code over $\mathbb{Z}_{4}$, but the conditions in his theorem involve finding the ranks of matrices having quite complicated entries, so that it is hard to verify the conditions without the help of a computer. Tanabe 36 then presented a simpler version of his theorem, and we can easily check its conditions by hand for the lifted Golay code over $\mathbb{Z}_{4}$.

2010 Mathematics Subject Classification. 05E30, 94B05, $05 \mathrm{~B} 05$.

Key words and phrases. Assmus-Mattson theorem, code, design, association scheme, Terwilliger algebra, multivariable polynomial interpolation. 
To be somewhat concrete, by an Assmus-Mattson-type theorem, we mean in this paper a theorem which enables us to find $t$-designs by just looking at some kind of weight enumerator of a code (plus a bit of extra information in some cases, e.g., linearity). Such a theorem is not always the best way to estimate the parameter $t$ of the resulting designs as it does not take into account the structure of the code at all (cf. Remark 5.5), but instead it has a great advantage in its wide range of applicability.

When working with the Hamming weight enumerator as in Theorem 1.1 we are dealing with codes in the Hamming association schemes. (Formal definitions will begin in Section 2, Hamming association schemes are examples of metric and cometric association schemes, and Theorem 1.1 can be interpreted and generalized from this point of view; cf. [37]. On the other hand, in situations where we focus on a more complicated type of weight enumerator of a block code as in [34, 36], we think of the code in question (say, of length $n$ ) as lying in a structure much finer than a Hamming association scheme; that is to say, the alphabet itself naturally becomes the vertex set of a commutative association scheme with $s$ classes where $s \geqslant 2$, and we consider its extension of length $n$. Hamming association schemes are the same thing as extensions of 1-class (i.e., trivial) association schemes, but if $s \geqslant 2$ then its extensions are no longer metric nor cometric.

In this paper, we prove a general Assmus-Mattson-type theorem for codes in extensions of arbitrary commutative association schemes. Our main results are Theorem 3.1 and Supplements 3.2 3.4. In general, the weights of a code take the form $\alpha=\left(\alpha_{1}, \alpha_{2}, \ldots, \alpha_{s}\right)$, where the $\alpha_{i}$ are non-negative integers such that $\sum_{i=1}^{s} \alpha_{i} \leqslant n$. We count the number of weights in a given interval when $s=1$ as in Theorem 1.1 but if $s \geqslant 2$ then instead we speak of the minimal degree of subspaces of the polynomial ring $\mathbb{R}\left[\xi_{1}, \xi_{2}, \ldots, \xi_{s}\right]$ which allow unique Lagrange interpolation with respect to those weights (which are lattice points in $\mathbb{R}^{s}$ ) contained in a given region. When specialized to the case of $\mathbb{Z}_{4}$-linear codes with the symmetrized weight enumerator as in [34, 36], the association scheme on the alphabet $\mathbb{Z}_{4}$ has 2 classes $R_{1}$ and $R_{2}$, together with the identity class $R_{0}$, defined by

$$
(x, y) \in R_{i} \Longleftrightarrow y-x= \pm i(\bmod 4) \quad\left(x, y \in \mathbb{Z}_{4}\right)
$$

for $i \in\{0,1,2\}$, and our results give a slight extension of Tanabe's theorem in [36]. The Assmus-Mattson-type theorem for $\mathbb{Z}_{4}$-linear codes with the Hamming weight enumerator due to Shin, Kumar, and Helleseth [30] can also be recovered. To prove our results, we make heavy use of the representation theory of the Terwilliger algebra 40, 41, 42, which is a non-commutative semisimple matrix $\mathbb{C}$-algebra attached to each vertex of an association scheme. See, e.g., [29, 15, 37, 3] for more applications of the Terwilliger algebra to coding theory and design theory.

The layout of this paper is as follows. Section 2 collects necessary notation, definitions, and results concerning commutative association schemes. In Section 3 . we state our main results. Section 4 is devoted to their proofs. Finally, we discuss a number of examples in Section 5 .

\section{Preliminaries}

We refer the reader to [12, 4, 9, 13, 25] for more background information. In this paper, $\mathbb{N}$ will denote the set of non-negative integers:

$$
\mathbb{N}=\{0,1,2, \ldots\} .
$$


2.1. Commutative association schemes and their Terwilliger algebras. Let $X$ be a finite set, and let $V$ be a complex vector space with a distinguished basis $\{\hat{x}: x \in X\}$ and a Hermitian inner product $\langle\hat{x}, \hat{y}\rangle=\delta_{x y}(x, y \in X)$. For every subset $C$ of $X$, we let $\hat{C}=\sum_{x \in C} \hat{x} \in V$ denote its characteristic vector. We will naturally identify $\operatorname{End}(V)$ with the $\mathbb{C}$-algebra of complex matrices with rows and columns indexed by $X$. The adjoint (or conjugate-transpose) of $A \in \operatorname{End}(V)$ will be denoted by $A^{\dagger}$. Let $\mathcal{R}=\left\{R_{0}, R_{1}, \ldots, R_{s}\right\}$ be a set of non-empty binary relations on $X$. For each $i$, let $A_{i} \in \operatorname{End}(V)$ be the $0-1$ adjacency matrix of the graph $\left(X, R_{i}\right)$ (directed, in general). The pair $(X, \mathcal{R})$ is called a commutative association scheme with $s$ classes if

(AS1) $A_{0}=I$, the identity matrix;

(AS2) $\sum_{i=0}^{s} A_{i}=J$, the all ones matrix;

(AS3) $A_{i}^{\dagger} \in\left\{A_{0}, A_{1}, \ldots, A_{s}\right\}$ for $0 \leqslant i \leqslant s$

(AS4) $A_{i} A_{j}=A_{j} A_{i} \in M:=\sum_{k=0}^{s} \mathbb{C} A_{k}$ for $0 \leqslant i, j \leqslant s$.

For the rest of this paper, we will always assume that $(X, \mathcal{R})$ is a commutative association scheme with $s$ classes. It follows from (AS1), (AS2), and (AS4) that the linear subspace $M$ of $\operatorname{End}(V)$ is an $(s+1)$-dimensional commutative $\mathbb{C}$-algebra, called the Bose-Mesner algebra of $(X, \mathcal{R})$. By (A, 3$), M$ is closed under ${ }^{\dagger}$, so that it is semisimple and has a basis $\left\{E_{i}\right\}_{i=0}^{s}$ consisting of the primitive idempotents, i.e., $E_{i} E_{j}=\delta_{i j} E_{i}, \sum_{i=0}^{s} E_{i}=I$. We will always set

$$
E_{0}=|X|^{-1} J
$$

We note that the $E_{i}$ are Hermitian positive semidefinite matrices. By (AS2), $M$ is also closed under entrywise (or Hadamard or Schur) multiplication, denoted o. The $A_{i}$ are the primitive idempotents of $M$ with respect to this multiplication, i.e., $A_{i} \circ A_{j}=\delta_{i j} A_{i}, \sum_{i=0}^{s} A_{i}=J$.

The intersection numbers $p_{i j}^{k}$ and the Krein parameters $q_{i j}^{k}(0 \leqslant i, j, k \leqslant s)$ of $(X, \mathcal{R})$ are defined by the equations

$$
A_{i} A_{j}=\sum_{k=0}^{s} p_{i j}^{k} A_{k}, \quad E_{i} \circ E_{j}=|X|^{-1} \sum_{k=0}^{s} q_{i j}^{k} E_{k} .
$$

Clearly, the $p_{i j}^{k}$ are non-negative integers. On the other hand, since $E_{i} \circ E_{j}$ (being a principal submatrix of $E_{i} \otimes E_{j}$ ) is positive semidefinite, it follows that the $q_{i j}^{k}$ are real and non-negative.

The change-of-basis matrices $P$ and $Q$ are defined by

$$
A_{i}=\sum_{j=0}^{s} P_{j i} E_{j}, \quad E_{i}=|X|^{-1} \sum_{j=0}^{s} Q_{j i} A_{j} .
$$

In particular,

$$
P Q=Q P=|X| I
$$

We refer to $P$ and $Q$ as the first and the second eigenmatrix of $(X, \mathcal{R})$, respectively. Note that $P_{0 i}$ is the degree (both in and out) of the regular graph $\left(X, R_{i}\right)$, and that $Q_{0 i}$ is the rank of $E_{i}$. Moreover, we have

$$
P_{i 0}=Q_{i 0}=1 \quad(0 \leqslant i \leqslant s) .
$$


We recall the Terwilliger algebra. Fix a "base vertex" $x_{0} \in X$, and define the diagonal matrices $E_{i}^{*}=E_{i}^{*}\left(x_{0}\right), A_{i}^{*}=A_{i}^{*}\left(x_{0}\right)(0 \leqslant i \leqslant s)$ in $\operatorname{End}(V)$ by

$$
\left(E_{i}^{*}\right)_{x x}=\left(A_{i}\right)_{x_{0} x}, \quad\left(A_{i}^{*}\right)_{x x}=|X|\left(E_{i}\right)_{x_{0} x} \quad(x \in X) .
$$

Note that $E_{i}^{*} E_{j}^{*}=\delta_{i j} E_{i}^{*}, \sum_{i=0}^{s} E_{i}^{*}=I$, and moreover

$$
A_{i}^{*} A_{j}^{*}=\sum_{k=0}^{s} q_{i j}^{k} A_{k}^{*}, \quad A_{i}^{*}=\sum_{j=0}^{s} Q_{j i} E_{j}^{*} .
$$

The $E_{i}^{*}$ and the $A_{i}^{*}$ are called the dual idempotents and the dual adjacency matrices of $(X, \mathcal{R})$ with respect to $x_{0}$, respectively. They form two bases of the dual BoseMesner algebra $M^{*}=M^{*}\left(x_{0}\right)$ of $(X, \mathcal{R})$ with respect to $x_{0}$. The Terwilliger (or subconstituent) algebra $T=T\left(x_{0}\right)$ of $(X, \mathcal{R})$ with respect to $x_{0}$ is the $\mathbb{C}$-subalgebra of $\operatorname{End}(V)$ generated by $M$ and $M^{*}$ [40, 41, 42]. The following are relations in $T$ (cf. [40, Lemma 3.2]):

$$
E_{i}^{*} A_{j} E_{k}^{*}=0 \Longleftrightarrow p_{i j}^{k}=0 ; \quad E_{i} A_{j}^{*} E_{k}=0 \Longleftrightarrow q_{i j}^{k}=0 .
$$

Since $T$ is closed under ${ }^{\dagger}$, it is semisimple and any two non-isomorphic irreducible $T$-modules in the standard module $V$ are orthogonal. Define a partition

$$
X=X_{0} \sqcup X_{1} \sqcup \cdots \sqcup X_{s}
$$

by

$$
X_{i}=\left\{x \in X:\left(x_{0}, x\right) \in R_{i}\right\} \quad(0 \leqslant i \leqslant s) .
$$

Then, since $\hat{X}_{i}=A_{i}^{\dagger} \hat{x}_{0}=E_{i}^{*} \hat{X}$ for every $0 \leqslant i \leqslant s$, it is immediate to see that the $(s+1)$-dimensional subspace

$$
\sum_{i=0}^{s} \mathbb{C} \hat{X}_{i}=M \hat{x}_{0}=M^{*} \hat{X}
$$

is an irreducible $T$-module, called the primary $T$-module. It is the unique irreducible $T$-module in $V$ containing the 1-dimensional subspaces $E_{0} V$ and $E_{0}^{*} V$.

Let $C$ be a subset of $X$. To avoid triviality, we call $C$ a code if $1<|C|<|X|$. For the moment, assume that $C$ is a code. The inner distribution of $C$ is the vector $a=\left(a_{0}, a_{1}, \ldots, a_{s}\right) \in \mathbb{R}^{s+1}$ defined by

$$
a_{i}=|C|^{-1}\left\langle\hat{C}, A_{i} \hat{C}\right\rangle=|C|^{-1} \cdot\left|R_{i} \cap(C \times C)\right| \quad(0 \leqslant i \leqslant s) .
$$

Observe that (cf. (2), (3))

$$
a_{0}=1, \quad \sum_{i=0}^{s} a_{i}=|C|, \quad(a Q)_{0}=|C|, \quad \sum_{i=0}^{s}(a Q)_{i}=|X| .
$$

Clearly, the $a_{i}$ are non-negative. On the other hand, from (11) it follows that

$$
\left\langle\hat{C}, E_{i} \hat{C}\right\rangle=|X|^{-1}|C|(a Q)_{i} \quad(0 \leqslant i \leqslant s) .
$$

Since the $E_{i}$ are positive semidefinite, it follows that the $(a Q)_{i}$ are also non-negative. Delsarte's famous linear programming bound [12] on the sizes of codes is based on this simple observation. The vector $a Q \in \mathbb{R}^{s+1}$ is often referred to as the MacWilliams transform of $a$. We remark the following:

$$
(a Q)_{i}=0 \Longleftrightarrow E_{i} \hat{C}=0 .
$$


2.2. Translation association schemes. Suppose that $X$ is endowed with the structure of an abelian group (written additively) with identity element 0 . We call $(X, \mathcal{R})$ a translation association scheme $[9, \S 2.10]$ if for all $0 \leqslant i \leqslant s$ and $z \in X$, $(x, y) \in R_{i}$ implies $(x+z, y+z) \in R_{i}$.

For the rest of this section, assume that $(X, \mathcal{R})$ is a translation association scheme. In this context, we will always choose 0 as the base vertex. (Note that the automorphism group of $(X, \mathcal{R})$ is transitive on $X$.) Observe that

$$
R_{i}=\left\{(x, y) \in X \times X: y-x \in X_{i}\right\} \quad(0 \leqslant i \leqslant s) .
$$

Let $X^{*}$ be the character group of $X$ with identity element $\iota$. To each $\varepsilon \in X^{*}$ we associate the vector

$$
\hat{\varepsilon}=|X|^{-1 / 2} \sum_{x \in X} \overline{\varepsilon(x)} \hat{x} \in V,
$$

so that

$$
\langle\hat{x}, \hat{\varepsilon}\rangle=|X|^{-1 / 2} \varepsilon(x) \quad\left(x \in X, \varepsilon \in X^{*}\right) .
$$

Note that the $\hat{\varepsilon}$ form an orthonormal basis of $V$ by the orthogonality relations for the characters. Moreover, it follows that

$$
A_{i} \hat{\varepsilon}=\left(\sum_{x \in X_{i}} \overline{\varepsilon(x)}\right) \hat{\varepsilon} \quad\left(0 \leqslant i \leqslant s, \varepsilon \in X^{*}\right) .
$$

This shows that each of the $\hat{\varepsilon}$ is an eigenvector for $M$, and hence belongs to one of the $E_{i} V$. Thus, we have a partition

$$
X^{*}=X_{0}^{*} \sqcup X_{1}^{*} \sqcup \cdots \sqcup X_{s}^{*},
$$

given by

$$
X_{i}^{*}=\left\{\varepsilon \in X^{*}: \hat{\varepsilon} \in E_{i} V\right\} \quad(0 \leqslant i \leqslant s) .
$$

Note that $X_{0}^{*}=\{\iota\}$, and that

$$
E_{i}=\sum_{\varepsilon \in X_{i}^{*}} \hat{\varepsilon} \hat{\varepsilon}^{\dagger} \quad(0 \leqslant i \leqslant s) .
$$

Define the set $\mathcal{R}^{*}=\left\{R_{0}^{*}, R_{1}^{*}, \ldots, R_{s}^{*}\right\}$ of non-empty binary relations on $X^{*}$ by

$$
R_{i}^{*}=\left\{(\varepsilon, \eta) \in X^{*} \times X^{*}: \eta \varepsilon^{-1} \in X_{i}^{*}\right\} \quad(0 \leqslant i \leqslant s) .
$$

Then it follows from the orthogonality relations and (8) that

$$
A_{i}^{*}=\sum_{(\varepsilon, \eta) \in R_{i}^{*}} \hat{\varepsilon} \hat{\eta}^{\dagger} \quad(0 \leqslant i \leqslant s) .
$$

In other words, the matrix representing $A_{i}^{*}$ with respect to the orthonormal basis $\left\{\hat{\varepsilon}: \varepsilon \in X^{*}\right\}$ of $V$ is precisely the $0-1$ adjacency matrix of the graph $\left(X^{*}, R_{i}^{*}\right)$. It turns out that the pair $\left(X^{*}, \mathcal{R}^{*}\right)$ is again a translation association scheme, called the dual of $(X, \mathcal{R})$. In particular, the $q_{i j}^{k}$ are the intersection numbers of $\left(X^{*}, \mathcal{R}^{*}\right)$, so that these are again non-negative integers in this case. We also note that $\left(X^{*}, \mathcal{R}^{*}\right)$ has eigenmatrices $P^{*}=Q$ and $Q^{*}=P$, and that

$$
P_{j i}=\sum_{x \in X_{i}} \overline{\varepsilon(x)}\left(\varepsilon \in X_{j}^{*}\right), \quad Q_{j i}=\sum_{\varepsilon \in X_{i}^{*}} \varepsilon(x) \quad\left(x \in X_{j}\right) .
$$

We will view $V$ together with the basis $\left\{\hat{\varepsilon}: \varepsilon \in X^{*}\right\}$ as the standard module for $\left(X^{*}, \mathcal{R}^{*}\right)$, and choose $\iota$ as the base vertex. 
A code $C$ in $X$ is called an additive code if it is a subgroup of $X$. Assume for the moment that $C$ is an additive code, and let $a=\left(a_{0}, a_{1}, \ldots, a_{s}\right)$ be its inner distribution. Observe that

$$
a_{i}=\left|X_{i} \cap C\right| \quad(0 \leqslant i \leqslant s),
$$

and hence $a$ is also called the weight distribution of $C$ in this case. The dual code of $C$ is the subgroup $C^{\perp}$ in $X^{*}$ defined by

$$
C^{\perp}=\left\{\varepsilon \in X^{*}: \varepsilon(x)=1 \text { for all } x \in C\right\} .
$$

From (7) it follows that

$$
\hat{C}=|X|^{-1 / 2}|C| \sum_{\varepsilon \in C^{\perp}} \hat{\varepsilon} .
$$

In other words, $\hat{C}$ is a scalar multiple of the characteristic vector of $C^{\perp}$ with respect to the basis $\left\{\hat{\varepsilon}: \varepsilon \in X^{*}\right\}$. We now observe that

$$
\left\langle\hat{C}, E_{i} \hat{C}\right\rangle=|X|^{-1}|C|^{2} \cdot\left|X_{i}^{*} \cap C^{\perp}\right| \quad(0 \leqslant i \leqslant s) .
$$

In particular, combining this with (6), we have

$$
\left|X_{i}^{*} \cap C^{\perp}\right|=|C|^{-1}(a Q)_{i} \quad(0 \leqslant i \leqslant s),
$$

so that $|C|^{-1}(a Q)$ gives the weight distribution of $C^{\perp}$.

The group operation on $X^{*}$ is multiplicative. In many cases (cf. Section 5), we fix a (non-canonical) isomorphism $X \rightarrow X^{*}\left(x \mapsto \varepsilon_{x}\right)$ such that

$$
\varepsilon_{x}(y)=\varepsilon_{y}(x) \quad(x, y \in X) .
$$

Then the dual code of an additive code in $X$ becomes again an additive code in $X$.

See [12, Chapter 6], [9, §2.10], and [25, §6] for more details about translation association schemes.

2.3. Extensions of commutative association schemes and Hamming association schemes. For the rest of this paper, we will fix an integer $n$ at least 2 . Delsarte [12, §2.5] gave a construction of a new commutative association scheme from $(X, \mathcal{R})$ with vertex set $X^{n}$ as follows. For a sequence $\alpha=\left(\alpha_{1}, \alpha_{2}, \ldots, \alpha_{s}\right) \in \mathbb{N}^{s}$, let $|\alpha|=\sum_{i=1}^{s} \alpha_{i}$. For any two vertices $\boldsymbol{x}=\left(x_{1}, x_{2}, \ldots, x_{n}\right), \boldsymbol{y}=\left(y_{1}, y_{2}, \ldots, y_{n}\right) \in X^{n}$, define the composition of $\boldsymbol{x}, \boldsymbol{y}$ to be the vector $c(\boldsymbol{x}, \boldsymbol{y})=\left(c_{1}, c_{2}, \ldots, c_{s}\right) \in \mathbb{N}^{s}$, where

$$
c_{i}=\left|\left\{\ell:\left(x_{\ell}, y_{\ell}\right) \in R_{i}\right\}\right| \quad(1 \leqslant i \leqslant s) .
$$

It is clear that $|c(\boldsymbol{x}, \boldsymbol{y})| \leqslant n$. For every $\alpha \in \mathbb{N}^{s}$ with $|\alpha| \leqslant n$, define the binary relation $\boldsymbol{R}_{\alpha}$ on $X^{n}$ by

$$
\boldsymbol{R}_{\alpha}=\left\{(\boldsymbol{x}, \boldsymbol{y}) \in X^{n} \times X^{n}: c(\boldsymbol{x}, \boldsymbol{y})=\alpha\right\} .
$$

Let

$$
\operatorname{Sym}^{n}(\mathcal{R})=\left\{\boldsymbol{R}_{\alpha}: \alpha \in \mathbb{N}^{s},|\alpha| \leqslant n\right\} .
$$

Then it follows that the pair $\left(X^{n}, \operatorname{Sym}^{n}(\mathcal{R})\right)$ is a commutative association scheme, called the extension of $(X, \mathcal{R})$ of length $n$. We will identify its standard module with $V^{\otimes n}$, so that $\hat{\boldsymbol{x}}:=\hat{x}_{1} \otimes \hat{x}_{2} \otimes \cdots \otimes \hat{x}_{n}$ for $\boldsymbol{x}=\left(x_{1}, x_{2}, \ldots, x_{n}\right) \in X^{n}$. For every $\alpha=\left(\alpha_{1}, \alpha_{2}, \ldots, \alpha_{s}\right) \in \mathbb{N}^{s}$ with $|\alpha| \leqslant n$, the 0 - 1 adjacency matrix $\boldsymbol{A}_{\alpha} \in \operatorname{End}\left(V^{\otimes n}\right)$ of the graph $\left(X^{n}, \boldsymbol{R}_{\alpha}\right)$ is then given by

$$
\boldsymbol{A}_{\alpha}=\sum_{i_{1}, i_{2}, \ldots, i_{n}} A_{i_{1}} \otimes A_{i_{2}} \otimes \cdots \otimes A_{i_{n}},
$$


where the sum is over $i_{1}, i_{2}, \ldots, i_{n} \in \mathbb{N}$ such that

$$
\left\{i_{1}, i_{2}, \ldots, i_{n}\right\}=\left\{0^{n-|\alpha|}, 1^{\alpha_{1}}, 2^{\alpha_{2}}, \ldots, s^{\alpha_{s}}\right\}
$$

as multisets. In particular, the Bose-Mesner algebra $M$ of $\left(X^{n}, \operatorname{Sym}^{n}(\mathcal{R})\right)$ coincides with the $n^{\text {th }}$ symmetric tensor space of $M$. Similar expressions hold for the primitive idempotents, dual idempotents, and the dual adjacency matrices of $\left(X^{n}, \operatorname{Sym}^{n}(\mathcal{R})\right)$, denoted henceforth by the $\boldsymbol{E}_{\alpha}, \boldsymbol{E}_{\alpha}^{*}$, and the $\boldsymbol{A}_{\alpha}^{*}$, respectively. For simplicity, we will always choose $\boldsymbol{x}_{0}:=\left(x_{0}, x_{0}, \ldots, x_{0}\right) \in X^{n}$ as the base vertex. We denote the corresponding dual Bose-Mesner algebra and the Terwilliger algebra by $\boldsymbol{M}^{*}$ and $\boldsymbol{T}$, respectively. We also consider the partition

$$
X^{n}=\bigsqcup_{\substack{\alpha \in \mathbb{N}^{s} \\|\alpha| \leqslant n}}\left(X^{n}\right)_{\alpha}
$$

corresponding to (5), i.e.,

$$
\left(X^{n}\right)_{\alpha}=\left\{\boldsymbol{x} \in X^{n}:\left(\boldsymbol{x}_{0}, \boldsymbol{x}\right) \in \boldsymbol{R}_{\alpha}\right\} .
$$

Let $\left\{\boldsymbol{e}_{i}: 1 \leqslant i \leqslant s\right\}$ be the standard basis of $\mathbb{R}^{s}$. Then in view of (3), we have

$$
\boldsymbol{A}_{\boldsymbol{e}_{i}}=\sum_{\substack{\alpha \in \mathbb{N}^{s} \\|\alpha| \leqslant n}}\left(\sum_{j=0}^{s} \alpha_{j} P_{j i}\right) \boldsymbol{E}_{\alpha}, \quad \boldsymbol{A}_{\boldsymbol{e}_{i}}^{*}=\sum_{\substack{\alpha \in \mathbb{N}^{s} \\|\alpha| \leqslant n}}\left(\sum_{j=0}^{s} \alpha_{j} Q_{j i}\right) \boldsymbol{E}_{\alpha}^{*},
$$

where $\alpha_{0}:=n-|\alpha|$. More generally, Mizukawa and Tanaka 27] described the eigenmatrices of $\left(X^{n}, \operatorname{Sym}^{n}(\mathcal{R})\right)$ in terms of certain $s$-variable hypergeometric orthogonal polynomials which generalize the Krawtchouk polynomials. See also [22, 21. Let $\boldsymbol{p}_{\alpha \beta}^{\gamma}$ (resp. $\boldsymbol{q}_{\alpha \beta}^{\gamma}$ ) denote the intersection numbers (resp. Krein parameters) of $\left(X^{n}, \operatorname{Sym}^{n}(\mathcal{R})\right)$. Then, for all $1 \leqslant i \leqslant s$ and $\beta, \gamma \in \mathbb{N}^{s}$ with $|\beta|,|\gamma| \leqslant n$, we have

$$
\boldsymbol{p}_{\boldsymbol{e}_{i} \beta}^{\gamma} \neq 0 \Longleftrightarrow \gamma \in\left\{\beta-\boldsymbol{e}_{j}+\boldsymbol{e}_{k}: p_{i j}^{k} \neq 0\right\},
$$

where we set $\boldsymbol{e}_{0}:=0$. A similar result holds for the $\boldsymbol{q}_{\boldsymbol{e}_{i} \beta}^{\gamma}$.

Let $\xi=\left(\xi_{0}, \xi_{1}, \ldots, \xi_{s}\right)$ be a sequence of $s+1$ variables. For every $\alpha \in \mathbb{N}^{s}$ with $|\alpha| \leqslant n$, we let

$$
\xi^{\alpha}=\xi_{0}^{n-|\alpha|} \xi_{1}^{\alpha_{1}} \xi_{2}^{\alpha_{2}} \ldots \xi_{s}^{\alpha_{s}} .
$$

Then it follows from (12) that

$$
\left(\sum_{i=0}^{s} \xi_{i} A_{i}\right)^{\otimes n}=\sum_{\substack{\alpha \in \mathbb{N}^{s} \\|\alpha| \leqslant n}} \xi^{\alpha} \boldsymbol{A}_{\alpha}
$$

and similarly for the $\boldsymbol{E}_{\alpha}$. Observe that

$$
\sum_{i=0}^{s} \xi_{i} E_{i}=|X|^{-1} \sum_{i=0}^{s}\left(\xi Q^{\top}\right)_{i} A_{i} .
$$

Combining these comments, we have (cf. [39, 16])

$$
\sum_{\substack{\alpha \in \mathbb{N}^{s} \\|\alpha| \leqslant n}} \xi^{\alpha} \boldsymbol{E}_{\alpha}=|X|^{-n} \sum_{\substack{\alpha \in \mathbb{N}^{s} \\|\alpha| \leqslant n}}\left(\xi Q^{\top}\right)^{\alpha} \boldsymbol{A}_{\alpha} .
$$

(Here, we extend the notation (15) to the sequence $\xi Q^{\top}$ as well.) 
Now, let $C$ be a code in $X^{n}$ with inner distribution $a=\left(a_{\alpha}\right)_{\alpha \in \mathbb{N}^{s},|\alpha| \leqslant n}$. Consider the polynomial $w_{C}(\xi)$ in $\mathbb{R}[\xi]=\mathbb{R}\left[\xi_{0}, \xi_{1}, \ldots, \xi_{s}\right]$ defined by

$$
w_{C}(\xi)=\sum_{\substack{\alpha \in \mathbb{N}^{s} \\|\alpha| \leqslant n}} a_{\alpha} \xi^{\alpha} .
$$

Note that $w_{C}(\xi)$ is homogeneous of degree $n$. From (16) it follows that

$$
|C|^{-1} \sum_{\substack{\alpha \in \mathbb{N}^{s} \\|\alpha| \leqslant n}}\left\langle\hat{C}, \boldsymbol{E}_{\alpha} \hat{C}\right\rangle \xi^{\alpha}=|X|^{-n} w_{C}\left(\xi Q^{\top}\right) .
$$

Hence we can read which of the $\boldsymbol{E}_{\alpha} \hat{C}$ vanish from the expansion of $w_{C}\left(\xi Q^{\top}\right)$.

Suppose for the moment that $(X, \mathcal{R})$ is a translation association scheme, and that $C$ is an additive code in $X^{n}$. In this case, $w_{C}(\xi)$ is called the weight enumerator of $C$. It should be remarked that $\left(X^{n}, \operatorname{Sym}^{n}(\mathcal{R})\right)$ and $\left(X^{* n}, \operatorname{Sym}^{n}\left(\mathcal{R}^{*}\right)\right)$ are dual to each other. By (10) and (17) we have (cf. [16])

$$
w_{C^{\perp}}(\xi)=|C|^{-1} w_{C}\left(\xi Q^{\top}\right) .
$$

This generalizes the well-known MacWilliams identity.

In proving our results, we also need to consider a special fusion of $\left(X^{n}, \operatorname{Sym}^{n}(\mathcal{R})\right)$ called the Hamming association scheme $H(n,|X|)$, which is defined to be the extension of length $n$ of the 1-class association scheme $\left(X,\left\{R_{0},(X \times X) \backslash R_{0}\right\}\right)$. Observe that $H(n,|X|)$ has $n$ classes, and that the associated matrices as well as the partition of the vertex set $X^{n}$ are parametrized by the integers $0,1, \ldots, n$, i.e., $\boldsymbol{A}_{i}, \boldsymbol{E}_{i}, \boldsymbol{E}_{i}^{*}, \boldsymbol{A}_{i}^{*}$, and also $\left(X^{n}\right)_{i}(0 \leqslant i \leqslant n)$. We denote the corresponding Bose-Mesner algebra, the dual Bose-Mesner algebra, and the Terwilliger algebra by $\boldsymbol{M}_{H}, \boldsymbol{M}_{H}^{*}$, and $\boldsymbol{T}_{H}$, respectively. Note that

$$
\boldsymbol{A}_{1}=\sum_{i=0}^{n} \theta_{i} \boldsymbol{E}_{i}, \quad \boldsymbol{A}_{1}^{*}=\sum_{i=0}^{n} \theta_{i}^{*} \boldsymbol{E}_{i}^{*}
$$

where

$$
\theta_{i}=\theta_{i}^{*}=n(|X|-1)-|X| i \quad(0 \leqslant i \leqslant n) .
$$

Below we collect important facts about the irreducible $\boldsymbol{T}_{H}$-modules, most of which can be found in Terwilliger's lecture notes [44. See also [38, §5.1]. (Some of the results hold in the wider class of metric and cometric association schemes.)

Lemma 2.1. Let $W$ be an irreducible $\boldsymbol{T}_{H}$-module.

(i) $\boldsymbol{A}_{1} \boldsymbol{E}_{i}^{*} W \subset \boldsymbol{E}_{i-1}^{*} W+\boldsymbol{E}_{i}^{*} W+\boldsymbol{E}_{i+1}^{*} W(0 \leqslant i \leqslant n)$, where $\boldsymbol{E}_{-1}^{*}=\boldsymbol{E}_{n+1}^{*}=0$.

(ii) $\boldsymbol{A}_{1}^{*} \boldsymbol{E}_{i} W \subset \boldsymbol{E}_{i-1} W+\boldsymbol{E}_{i} W+\boldsymbol{E}_{i+1} W(0 \leqslant i \leqslant n)$, where $\boldsymbol{E}_{-1}=\boldsymbol{E}_{n+1}=0$.

(iii) There are non-negative integers $r$ and $d$ such that

$$
n-2 r \leqslant d \leqslant n-r,
$$

and

$$
\operatorname{dim} \boldsymbol{E}_{i}^{*} W=\operatorname{dim} \boldsymbol{E}_{i} W=\left\{\begin{array}{ll}
1 & \text { if } r \leqslant i \leqslant r+d, \\
0 & \text { otherwise, }
\end{array} \quad(0 \leqslant i \leqslant n) .\right.
$$

(iv) $\boldsymbol{E}_{i}^{*} \boldsymbol{A}_{1} \boldsymbol{E}_{j}^{*} W \neq 0$ if $|i-j|=1 \quad(r \leqslant i, j \leqslant r+d)$.

(v) $\boldsymbol{E}_{i} \boldsymbol{A}_{1}^{*} \boldsymbol{E}_{j} W \neq 0$ if $|i-j|=1 \quad(r \leqslant i, j \leqslant r+d)$. 
The integers $r$ and $d$ in (iii) above are called the endpoint and the diameter of $W$, respectively. The integer $2 r+d-n$ is called the displacement [43] of $W$. From (19) it follows that

$$
0 \leqslant 2 r+d-n \leqslant n .
$$

For every $0 \leqslant c \leqslant n$, let $U_{c}$ be the span of the irreducible $\boldsymbol{T}_{H}$-modules in $V^{\otimes n}$ with displacement $c$. Then we have

$$
V^{\otimes n}=\bigoplus_{c=0}^{n} U_{c} .
$$

This is called the displacement decomposition of $V^{\otimes n}$. Terwilliger [44] showed that

$$
U_{0}=\left(\mathbb{C} \hat{x}_{0}+\mathbb{C} \hat{X}\right)^{\otimes n} .
$$

\section{MAin Results}

We recall some concepts from polynomial interpolation; cf. [14]. Let $S$ be a finite set of points in $\mathbb{R}^{s}$. A linear subspace $\mathscr{L}$ of the polynomial ring $\mathbb{R}\left[\xi_{1}, \xi_{2}, \ldots, \xi_{s}\right]$ is called an interpolation space with respect to $S$ if, for every $f \in \mathbb{R}\left[\xi_{1}, \xi_{2}, \ldots, \xi_{s}\right]$, there exists a unique $g \in \mathscr{L}$ such that $f(z)=g(z)$ for all $z=\left(z_{1}, z_{2}, \ldots, z_{s}\right) \in S$. It is called a minimal degree interpolation space if, moreover, this $g$ always satisfies $\operatorname{deg} f \geqslant \operatorname{deg} g$.

Let $\mathscr{M}(S)$ denote a minimal degree interpolation space with respect to $S$, and let

$$
\mu(S)=\max \{\operatorname{deg} f: f \in \mathscr{M}(S)\} .
$$

We note that $\mathscr{M}(S)$ exists; see Theorem 3.5 below. Observe also that $\mu(S)$ is well-defined, i.e., it is independent of the choice of $\mathscr{M}(S)$.

We retain the notation of Section 2, For $\boldsymbol{x}=\left(x_{1}, x_{2}, \ldots, x_{n}\right) \in X^{n}$, let

$$
\operatorname{supp}(\boldsymbol{x})=\left\{\ell: x_{\ell} \neq x_{0}\right\} \subset\{1,2, \ldots, n\} .
$$

We call $\operatorname{supp}(\boldsymbol{x})$ the support of $\boldsymbol{x}$ (with respect to $\boldsymbol{x}_{0}=\left(x_{0}, x_{0}, \ldots, x_{0}\right)$ ).

Theorem 3.1. Let $C$ be a code in $X^{n}$. Let

$$
S_{r}=\left\{\alpha \in \mathbb{N}^{s}: r \leqslant|\alpha| \leqslant n-r, \boldsymbol{E}_{\alpha}^{*} \hat{C} \neq 0\right\} \quad(1 \leqslant r \leqslant\lfloor n / 2\rfloor),
$$

and let

$$
\delta^{*}=\min \left\{i \neq 0: \boldsymbol{E}_{i} \hat{C} \neq 0\right\} .
$$

Suppose that an integer $t(1 \leqslant t \leqslant n)$ is such that

$$
\mu\left(S_{r}\right)<\delta^{*}-r \quad(1 \leqslant r \leqslant t) .
$$

Then the multiset

$$
\left\{\operatorname{supp}(\boldsymbol{x}): \boldsymbol{x} \in\left(X^{n}\right)_{\alpha} \cap C\right\}
$$

is a $t$-design (with block size $|\alpha|$ ) for every $\alpha \in \mathbb{N}^{s}$ with $|\alpha| \leqslant n$.

We use Theorem 3.1 together with the following "supplements".

Supplement 3.2. Let $C$ be a code in $X^{n}$. Assume that we are given in advance a set $K \subset \mathbb{N}^{s}$ such that the multiset (22) is a t-design for every $\alpha \in K$. Then the condition (21) in Theorem 3.1 may be replaced by

$$
\mu\left(S_{r} \backslash K\right)<\delta^{*}-r \quad(1 \leqslant r \leqslant t) .
$$


We call a subset $C$ of $X^{n}$ a weakly t-balanced array 1 over $(X, \mathcal{R})$ (with respect to $\left.\boldsymbol{x}_{0}\right)$ if, for any $\Lambda \subset\{1,2, \ldots, n\}$ and $\gamma \in \mathbb{N}^{s}$ such that $|\gamma| \leqslant|\Lambda| \leqslant t$, the number

$$
\left|\left\{\boldsymbol{x} \in C:\left(x_{\ell}\right)_{\ell \in \Lambda} \in\left(X^{|\Lambda|}\right)_{\gamma}\right\}\right|
$$

depends only on $|\Lambda|$ and $\gamma$.

Recall that, when considering a translation association scheme, we always choose the identity as the base vertex.

Supplement 3.3. Suppose that $(X, \mathcal{R})$ is a translation association scheme, and that $C$ is an additive code in $X^{n}$. Assume that we are given in advance a set $L \subset \mathbb{N}^{s}$ such that, for every $\alpha \in L,\left(X^{* n}\right)_{\alpha} \cap C^{\perp}$ is a weakly t-balanced array over $\left(X^{*}, \mathcal{R}^{*}\right)$. Then the scalar $\delta^{*}$ in Theorem 3.1 may be replaced by

$$
\min \left\{|\alpha|: 0 \neq \alpha \in \mathbb{N}^{s} \backslash L, \boldsymbol{E}_{\alpha} \hat{C} \neq 0\right\} .
$$

We note that, in the particular case where $\alpha \in \mathbb{N}^{s}$ is of the form $\alpha=h \boldsymbol{e}_{i}$ for some $h>0$, the condition that $\left(X^{* n}\right)_{\alpha} \cap C^{\perp}$ is a weakly $t$-balanced array over $\left(X^{*}, \mathcal{R}^{*}\right)$ is equivalent to saying that the multiset

$$
\left\{\operatorname{supp}(\varepsilon): \varepsilon \in\left(X^{* n}\right)_{\alpha} \cap C^{\perp}\right\}
$$

is a $t$-design.

Supplement 3.4 below was inspired by [36, Theorem 2], and allows us to estimate $\mu(S)$, and hence $t$, by geometrical considerations; see Section 5 It is a general result about minimal degree interpolation spaces, so that we give a proof right after the statement.

Supplement 3.4. Let $S$ be a finite set of points in $\mathbb{R}^{s}$. Suppose that there are real scalars $z_{i \ell}(1 \leqslant i \leqslant s, \ell \in \mathbb{N})$, a positive integer $m$, and a linear automorphism $\sigma \in \mathrm{GL}\left(\mathbb{R}^{s}\right)$ such that $z_{i k} \neq z_{i \ell}$ whenever $k \neq \ell$, and that

$$
\sigma(S) \subset\left\{\left(z_{1 \alpha_{1}}, z_{2 \alpha_{2}}, \ldots, z_{s \alpha_{s}}\right) \in \mathbb{R}^{s}: \alpha \in \mathbb{N}^{s},|\alpha| \leqslant m\right\} .
$$

Then $\mu(S) \leqslant m$.

Proof. We abbreviate $z_{\alpha}:=\left(z_{1 \alpha_{1}}, z_{2 \alpha_{2}}, \ldots, z_{s \alpha_{s}}\right)$. Let $\Sigma$ denote the RHS in (24). It suffices to show that $\mu(\Sigma) \leqslant m$. To this end, we construct an interpolation space with respect to $\Sigma$ with maximum degree at most $m$ as follows. Let $\alpha \in \mathbb{N}^{s}$ be given with $|\alpha| \leqslant m$, and assume that we have constructed polynomials

$$
f_{\beta} \in \mathbb{R}\left[\xi_{1}, \xi_{2}, \ldots, \xi_{s}\right] \quad\left(\beta \in \mathbb{N}^{s},|\alpha|<|\beta| \leqslant m\right)
$$

such that $\operatorname{deg} f_{\beta} \leqslant m$ and

$$
f_{\beta}\left(z_{\gamma}\right)=\delta_{\beta \gamma} \quad\left(\gamma \in \mathbb{N}^{s},|\gamma| \leqslant m\right) .
$$

Define $g_{\alpha} \in \mathbb{R}\left[\xi_{1}, \xi_{2}, \ldots, \xi_{s}\right]$ by

$$
g_{\alpha}=\prod_{i=1}^{s} \prod_{\ell=0}^{\alpha_{i}-1} \frac{\xi_{i}-z_{i \ell}}{z_{i \alpha_{i}}-z_{i \ell}},
$$

and let

$$
f_{\alpha}=g_{\alpha}-\sum_{\substack{\beta \in \mathbb{N}^{s} \\|\alpha|<|\beta| \leqslant m}} g_{\alpha}\left(z_{\beta}\right) f_{\beta} .
$$

\footnotetext{
${ }^{1}$ This term is meant as only provisional; cf. 33 .
} 
Then $\operatorname{deg} f_{\alpha} \leqslant m$, and it is easy to see that

$$
f_{\alpha}\left(z_{\gamma}\right)=\delta_{\alpha \gamma} \quad\left(\gamma \in \mathbb{N}^{s},|\gamma| \leqslant m\right) .
$$

Thus, by induction we obtain polynomials $f_{\alpha}$ with $\operatorname{deg} f_{\alpha} \leqslant m$ satisfying (25) for all $\alpha \in \mathbb{N}^{s}$ with $|\alpha| \leqslant m$. It is clear that the subspace

$$
\sum_{\substack{\alpha \in \mathbb{N}^{s} \\|\alpha| \leqslant m}} \mathbb{R} f_{\alpha} \subset \mathbb{R}\left[\xi_{1}, \xi_{2}, \ldots, \xi_{s}\right]
$$

is an interpolation space with respect to $\Sigma$, and the proof is complete.

We end this section by recalling a construction of a minimal degree interpolation space $\mathscr{M}(S)$ due to de Boor and Ron [7, 8. See also [14, §3]. For every non-zero element $f=\sum_{i=0}^{\infty} f_{i}$ in the ring of formal power series $\mathbb{R}\left[\left[\xi_{1}, \xi_{2}, \ldots, \xi_{s}\right]\right]$ where $f_{i}$ is homogeneous of degree $i$, let

$$
f_{\downarrow}=f_{i_{0}},
$$

where $i_{0}=\min \left\{i: f_{i} \neq 0\right\}$. We conventionally set $0_{\downarrow}:=0$.

Theorem 3.5 ( $[7,8])$. Let $S$ be a finite set of points in $\mathbb{R}^{s}$. Let $\mathscr{E}$ be the subspace of $\mathbb{R}\left[\left[\xi_{1}, \xi_{2}, \ldots, \xi_{s}\right]\right]$ spanned by the exponential functions

$$
\exp \left(\sum_{i=1}^{s} z_{i} \xi_{i}\right) \quad\left(\left(z_{1}, z_{2}, \ldots, z_{s}\right) \in S\right) .
$$

Then the subspace

$$
\sum_{f \in \mathscr{E}} \mathbb{R} f_{\downarrow} \subset \mathbb{R}\left[\xi_{1}, \xi_{2}, \ldots, \xi_{s}\right]
$$

is a minimal degree interpolation space with respect to $S$.

Theorem 3.5 immediately leads to the following formula for $\mu(S)$ which is well suited for computer calculations:

Supplement 3.6. For every finite set $S$ of points in $\mathbb{R}^{s}$, the scalar $\mu(S)$ equals the smallest $m \in \mathbb{N}$ for which the polynomials

$$
\sum_{k=0}^{m}\left(\sum_{i=1}^{s} z_{i} \xi_{i}\right)^{k} \quad\left(\left(z_{1}, z_{2}, \ldots, z_{s}\right) \in S\right)
$$

are linearly independent.

(Note that we just discarded the irrelevant factors $1 /(k !)$ in the Taylor polynomials of these exponential functions.)

\section{Proofs}

We begin by proving a few preliminary lemmas. Recall the space $U_{0}$ spanned by the irreducible $\boldsymbol{T}_{H}$-modules in $V^{\otimes n}$ with displacement 0 . We let $\pi_{U_{0}}: V^{\otimes n} \rightarrow U_{0}$ denote the orthogonal projection onto $U_{0}$. Note that $\pi_{U_{0}}$ is a $\boldsymbol{T}_{H}$-homomorphism.

Lemma 4.1. The primary $\boldsymbol{T}$-module $\boldsymbol{M} \hat{\boldsymbol{x}}_{0}$ is orthogonal to every non-primary irreducible $\boldsymbol{T}_{H}$-module in $U_{0}$. 
Proof. Let $u_{0}=\hat{x}_{0}$ and $u_{1}=\hat{X}-\hat{x}_{0}$. For every $\tau=\left(\tau_{1}, \tau_{2}, \ldots, \tau_{n}\right) \in\{0,1\}^{n}$, let

$$
\boldsymbol{u}_{\tau}=u_{\tau_{1}} \otimes u_{\tau_{2}} \otimes \cdots \otimes u_{\tau_{n}} \in \boldsymbol{E}_{|\tau|}^{*} U_{0},
$$

where $|\tau|=\sum_{\ell=1}^{n} \tau_{\ell}$ denotes the weight of $\tau$. The $\boldsymbol{u}_{\tau}$ form an orthogonal basis of $U_{0}$ by (20), and we have

$$
\left\|\boldsymbol{u}_{\tau}\right\|^{2}=(|X|-1)^{|\tau|}
$$

For every $\alpha \in \mathbb{N}^{s}$ with $|\alpha| \leqslant n$, using (12) we routinely have

$$
\left\langle\boldsymbol{A}_{\alpha} \hat{\boldsymbol{x}}_{0}, \boldsymbol{u}_{\tau}\right\rangle=\delta_{|\tau|,|\alpha|}\left(\begin{array}{c}
|\alpha| \\
\alpha_{1}, \alpha_{2}, \ldots, \alpha_{s}
\end{array}\right) \prod_{i=1}^{s}\left(P_{0 i}\right)^{\alpha_{i}},
$$

where we recall that $P_{0 i}$ is the degree of $\left(X, R_{i}\right)$. Since

$$
\pi_{U_{0}} \boldsymbol{A}_{\alpha} \hat{\boldsymbol{x}}_{0}=\sum_{\tau \in\{0,1\}^{n}}\left\|\boldsymbol{u}_{\tau}\right\|^{-2}\left\langle\boldsymbol{A}_{\alpha} \hat{\boldsymbol{x}}_{0}, \boldsymbol{u}_{\tau}\right\rangle \boldsymbol{u}_{\tau}
$$

it follows from (26) and (27) that $\pi_{U_{0}} \boldsymbol{A}_{\alpha} \hat{\boldsymbol{x}}_{0}$ is a scalar multiple of

$$
\sum_{\substack{\tau \in\{0,1\}^{n} \\|\tau|=|\alpha|}} \boldsymbol{u}_{\tau}=\boldsymbol{A}_{|\alpha|} \hat{\boldsymbol{x}}_{0} \in \boldsymbol{M}_{H} \hat{\boldsymbol{x}}_{0} .
$$

It follows that $\pi_{U_{0}} \boldsymbol{M} \hat{\boldsymbol{x}}_{0}=\boldsymbol{M}_{H} \hat{\boldsymbol{x}}_{0}$, as desired.

Lemma 4.2. Let $C$ be a non-empty subset of $\left(X^{n}\right)_{k}$ for some $0 \leqslant k \leqslant n$. Then the following are equivalent:

(i) The multiset $\{\operatorname{supp}(\boldsymbol{x}): \boldsymbol{x} \in C\}$ is a t-design.

(ii) $\boldsymbol{E}_{i} \pi_{U_{0}} \hat{C}$ is a scalar multiple of $\boldsymbol{E}_{i} \hat{\boldsymbol{x}}_{0}$ for every $0 \leqslant i \leqslant t$.

(iii) $\hat{C}$ is orthogonal to every non-primary irreducible $\boldsymbol{T}_{H}$-module in $U_{0}$ with endpoint at most $t$.

Proof. First, we show (i) $\Leftrightarrow$ (ii). To this end, we introduce another orthogonal basis of $U_{0}$ as follows. Define $v_{0}, v_{1} \in V$ by

$$
v_{0}=E_{0} \hat{x}_{0}=|X|^{-1} \hat{X}, \quad v_{1}=\left(I-E_{0}\right) \hat{x}_{0}=\hat{x}_{0}-|X|^{-1} \hat{X} .
$$

Note that

$$
\left\|v_{0}\right\|^{2}=|X|^{-1}, \quad \|\left. v_{1}\right|^{2}=1-|X|^{-1}, \quad\left\langle v_{0}, v_{1}\right\rangle=0 .
$$

For every $\tau=\left(\tau_{1}, \tau_{2}, \ldots, \tau_{n}\right) \in\{0,1\}^{n}$, let

$$
\boldsymbol{v}_{\tau}=v_{\tau_{1}} \otimes v_{\tau_{2}} \otimes \cdots \otimes v_{\tau_{n}} \in \boldsymbol{E}_{|\tau|} U_{0}
$$

where $|\tau|=\sum_{\ell=1}^{n} \tau_{\ell}$. The $\boldsymbol{v}_{\tau}$ form an orthogonal basis of $U_{0}$ by (20), and we have

$$
\left\|\boldsymbol{v}_{\tau}\right\|^{2}=|X|^{-n}(|X|-1)^{|\tau|} .
$$

Moreover, observe that

$$
\sum_{\substack{\tau \in\{0,1\}^{n} \\|\tau|=i}} \boldsymbol{v}_{\tau}=\boldsymbol{E}_{i} \hat{\boldsymbol{x}}_{0}
$$

By these comments and since

$$
\boldsymbol{E}_{i} \pi_{U_{0}} \hat{C}=\sum_{\substack{\tau \in\{0,1\}^{n} \\|\tau|=i}}\left\|\boldsymbol{v}_{\tau}\right\|^{-2}\left\langle\hat{C}, \boldsymbol{v}_{\tau}\right\rangle \boldsymbol{v}_{\tau},
$$

it follows that (ii) holds if and only if $\left\langle\hat{C}, \boldsymbol{v}_{\tau}\right\rangle$ depends only on $|\tau|$ whenever $|\tau| \leqslant t$. 
Assume that (i) holds. Let $\tau \in\{0,1\}^{n}$ with $|\tau| \leqslant t$. From (28) it follows that

$$
\begin{aligned}
\left\langle\hat{C}, \boldsymbol{v}_{\tau}\right\rangle=|X|^{-n} \sum_{i=0}^{|\tau|}( & (-1)^{i}(|X|-1)^{|\tau|-i} \\
& \times|\{\boldsymbol{x} \in C:|\operatorname{supp}(\tau) \cap \operatorname{supp}(\boldsymbol{x})|=i\}|,
\end{aligned}
$$

which is indeed a constant depending only on $|\tau|$, and hence (ii) holds.

Conversely, assume that (ii) holds. Let $\tau \in\{0,1\}^{n}$ with $|\tau|=t$, and let

$$
\boldsymbol{w}_{\tau}=w_{\tau_{1}} \otimes w_{\tau_{2}} \otimes \cdots \otimes w_{\tau_{n}},
$$

where $w_{0}=\hat{X}=|X| v_{0}$ and $w_{1}=\hat{x}_{0}=v_{0}+v_{1}$. On the one hand, we have

$$
\left\langle\hat{C}, \boldsymbol{w}_{\tau}\right\rangle=|\{\boldsymbol{x} \in C: \operatorname{supp}(\tau) \cap \operatorname{supp}(\boldsymbol{x})=\emptyset\}| .
$$

On the other hand, observe that

$$
\boldsymbol{w}_{\tau}=|X|^{n-t} \sum_{\rho} \boldsymbol{v}_{\rho}
$$

where the sum is over $\rho \in\{0,1\}^{n}$ with $\operatorname{supp}(\rho) \subset \operatorname{supp}(\tau)$. It follows that the common value in (29) is independent of the choice of $\tau$, and hence (i) holds.

Next, we show (ii) $\Leftrightarrow$ (iii). Observe that

$$
\boldsymbol{E}_{i} U_{0}=\mathbb{C} \boldsymbol{E}_{i} \hat{\boldsymbol{x}}_{0} \perp \sum_{W} \boldsymbol{E}_{i} W \quad(0 \leqslant i \leqslant t),
$$

where the sum is over the non-primary irreducible $\boldsymbol{T}_{H}$-modules $W$ in $U_{0}$ with endpoint at most $i$. If (iii) holds, then the vectors $\boldsymbol{E}_{i} \hat{C}(0 \leqslant i \leqslant t)$ are also orthogonal to every non-primary irreducible $\boldsymbol{T}_{H}$-module in $U_{0}$ with endpoint at most $t$, and hence the vector $\pi_{U_{0}} \boldsymbol{E}_{i} \hat{C} \in \boldsymbol{E}_{i} U_{0}$ vanishes on the second term of the RHS in (30) for every $0 \leqslant i \leqslant t$; in other words, (ii) holds.

Conversely, let $W$ be a non-primary irreducible $\boldsymbol{T}_{H}$-module in $U_{0}$ with endpoint $r \leqslant t$, and assume that $\hat{C}$ is not orthogonal to $W$. Let $\pi_{W}: V^{\otimes n} \rightarrow W$ be the orthogonal projection onto $W$. Then we have $\pi_{W} \hat{C} \neq 0$. Let

$$
\ell=\min \left\{i: \boldsymbol{E}_{i} \pi_{W} \hat{C} \neq 0\right\} .
$$

By Lemma 2.1(iii), $\boldsymbol{E}_{\ell} \pi_{W} \hat{C}$ spans $\boldsymbol{E}_{\ell} W$. In view of Lemma 2.1(ii), (v), we have

$$
\boldsymbol{E}_{r}\left(\boldsymbol{A}_{1}^{*}\right)^{\ell-r} \pi_{W} \hat{C}=\boldsymbol{E}_{r}\left(\boldsymbol{A}_{1}^{*}\right)^{\ell-r} \boldsymbol{E}_{\ell} \pi_{W} \hat{C} \neq 0 .
$$

Since $\pi_{W}$ is a $\boldsymbol{T}_{H}$-homomorphism and since $\hat{C} \in \boldsymbol{E}_{k}^{*} V^{\otimes n}$, it follows from (18) that

$$
0 \neq \boldsymbol{E}_{r} \pi_{W}\left(\boldsymbol{A}_{1}^{*}\right)^{\ell-r} \hat{C}=\left(\theta_{k}^{*}\right)^{\ell-r} \boldsymbol{E}_{r} \pi_{W} \hat{C},
$$

i.e., we must have $\ell=r$. It follows that $\boldsymbol{E}_{i} \pi_{U_{0}} \hat{C}$ does not vanish on the second term of the RHS in (30) when $i=r$, and hence (ii) fails to hold. We have now shown (ii) $\Leftrightarrow$ (iii), and the proof is complete.

Lemma 4.3. Let $C$ be a non-empty subset of $\left(X^{n}\right)_{\alpha}$ for some $\alpha \in \mathbb{N}^{s}$ with $|\alpha| \leqslant n$. Suppose that $C$ is a weakly $t$-balanced array over $(X, \mathcal{R})$. Then

$$
\boldsymbol{E}_{i}^{*} \pi_{U_{0}} \boldsymbol{M} \hat{C}=\mathbb{C} \boldsymbol{A}_{i} \hat{\boldsymbol{x}}_{0} \quad(0 \leqslant i \leqslant t) .
$$


Proof. We fix $\beta \in \mathbb{N}^{s}$ with $|\beta| \leqslant n$, and consider the vector $\boldsymbol{A}_{\beta} \hat{C} \in \boldsymbol{M} \hat{C}$. We use the notation in the proof of Lemma 4.1. Let $\tau \in\{0,1\}^{n}$ with $|\tau| \leqslant t$. We will use ' and " to denote objects associated with the extensions of $(X, \mathcal{R})$ of lengths $|\tau|$ and $n-|\tau|$, respectively; e.g., $\boldsymbol{A}_{\gamma}^{\prime}\left(\gamma \in \mathbb{N}^{s},|\gamma| \leqslant|\tau|\right), \boldsymbol{A}_{i}^{\prime}(0 \leqslant i \leqslant|\tau|), \boldsymbol{x}_{0}^{\prime} \in X^{|\tau|}$ for the former. We understand that the coordinates of $X^{|\tau|}$ and $X^{n-|\tau|}$ are indexed by $\operatorname{supp}(\tau)$ and $\{1,2, \ldots, n\} \backslash \operatorname{supp}(\tau)$, respectively. With this notation established, we have

$$
\boldsymbol{A}_{\beta}=\sum_{\nu} \boldsymbol{A}_{\nu}^{\prime} \otimes \boldsymbol{A}_{\beta-\nu}^{\prime \prime}
$$

where the sum is over $\nu \in \mathbb{N}^{s}$ such that $\beta-\nu \in \mathbb{N}^{s},|\nu| \leqslant|\tau|$, and $|\beta-\nu| \leqslant n-|\tau|$. Observe also that

Hence we have

$$
\boldsymbol{u}_{\tau}=\boldsymbol{A}_{|\tau|}^{\prime} \hat{\boldsymbol{x}}_{0}^{\prime} \otimes \hat{\boldsymbol{x}}_{0}^{\prime \prime}
$$

$$
\begin{aligned}
\left\langle\boldsymbol{A}_{\beta} \hat{C}, \boldsymbol{u}_{\tau}\right\rangle & =\sum_{\nu, \rho} g_{\nu \rho} \cdot\left\langle\hat{C},\left(\boldsymbol{A}_{\rho}^{\prime}\right)^{\dagger} \hat{\boldsymbol{x}}_{0}^{\prime} \otimes\left(\boldsymbol{A}_{\beta-\nu}^{\prime \prime}\right)^{\dagger} \hat{\boldsymbol{x}}_{0}^{\prime \prime}\right\rangle \\
& =\sum_{\nu, \rho} g_{\nu \rho} \cdot\left|\left\{\boldsymbol{x} \in C:\left(x_{\ell}\right)_{\ell \in \operatorname{supp}(\tau)} \in\left(X^{|\tau|}\right)_{\rho}\right\}\right|,
\end{aligned}
$$

where the sums are over $\nu, \rho \in \mathbb{N}^{s}$ such that $|\nu|,|\rho| \leqslant|\tau|, \beta-\nu=\alpha-\rho \in \mathbb{N}^{s}$, and $|\beta-\nu| \leqslant n-|\tau|$, and where we write

$$
\boldsymbol{A}_{\nu}^{\prime} \boldsymbol{A}_{|\tau|}^{\prime}=\boldsymbol{A}_{|\tau|}^{\prime} \boldsymbol{A}_{\nu}^{\prime}=\sum_{\substack{\rho \in \mathbb{N}^{s} \\|\rho| \leqslant|\tau|}} g_{\nu \rho} \boldsymbol{A}_{\rho}^{\prime} .
$$

By the assumption, the RHS in (31) depends only on $|\tau| \leqslant t$, and hence it follows that $\boldsymbol{E}_{i}^{*} \pi_{U_{0}} \boldsymbol{A}_{\beta} \hat{C}$ is a scalar multiple of $\boldsymbol{A}_{i} \hat{\boldsymbol{x}}_{0}$ for every $0 \leqslant i \leqslant t$ as in the proof of Lemma 4.1. We have now shown that $\boldsymbol{E}_{i}^{*} \pi_{U_{0}} \boldsymbol{M} \hat{\boldsymbol{C}}$ is a subspace of $\mathbb{C} \boldsymbol{A}_{i} \hat{\boldsymbol{x}}_{0}$ for $0 \leqslant i \leqslant t$. That it is non-zero and hence agrees with $\mathbb{C} \boldsymbol{A}_{i} \hat{\boldsymbol{x}}_{0}$ follows from

$$
\boldsymbol{E}_{i}^{*} \pi_{U_{0}} J^{\otimes n} \hat{C}=|C| \boldsymbol{A}_{i} \hat{\boldsymbol{x}}_{0} .
$$

This completes the proof.

4.1. Proof of Theorem 3.1. Define $\boldsymbol{D}_{1}^{*}, \boldsymbol{D}_{2}^{*}, \ldots, \boldsymbol{D}_{s}^{*} \in \boldsymbol{M}^{*}$ by

$$
\boldsymbol{D}_{i}^{*}=\sum_{\substack{\alpha \in \mathbb{N}^{s} \\|\alpha| \leqslant n}} \alpha_{i} \boldsymbol{E}_{\alpha}^{*} \quad(1 \leqslant i \leqslant s) .
$$

Observe that the $\boldsymbol{D}_{i}^{*}$ generate $\boldsymbol{M}^{*}$. By (22), (3), and (13), for $1 \leqslant j \leqslant s$ we have

$$
\begin{aligned}
\sum_{i=1}^{s} P_{i j} \boldsymbol{A}_{\boldsymbol{e}_{i}}^{*} & =\sum_{\substack{\alpha \in \mathbb{N}^{s} \\
|\alpha| \leqslant n}}\left(\sum_{h=0}^{s} \alpha_{h} \sum_{i=1}^{s} Q_{h i} P_{i j}\right) \boldsymbol{E}_{\alpha}^{*} \\
& =\sum_{\substack{\alpha \in \mathbb{N}^{s} \\
|\alpha| \leqslant n}}\left(\sum_{h=0}^{s} \alpha_{h}\left(|X| \delta_{h j}-Q_{h 0} P_{0 j}\right)\right) \boldsymbol{E}_{\alpha}^{*} \\
& =|X| \boldsymbol{D}_{j}^{*}-n P_{0 j} I^{\otimes n},
\end{aligned}
$$

where we have used $\alpha_{0}=n-|\alpha|$. In particular, the $\boldsymbol{A}_{\boldsymbol{e}_{i}}^{*}$ also generate $\boldsymbol{M}^{*}$.

Now, fix $\alpha \in \mathbb{N}^{s}$ with $|\alpha| \leqslant n$. We invoke Lemma 4.2 to show that the multiset (22) is a $t$-design. Let $W$ be a non-primary irreducible $\boldsymbol{T}_{H}$-module in $U_{0}$ with 
endpoint $r \leqslant t$. Recall that $W$ has diameter $n-2 r$. It suffices to show that $\boldsymbol{E}_{\alpha}^{*} \hat{C}$ is orthogonal to $W$. Let $\pi_{W}: V^{\otimes n} \rightarrow W$ be the orthogonal projection onto $W$. First, we show that

$$
\pi_{W} \boldsymbol{E}_{\alpha}^{*} \hat{C} \in \sum_{i=\delta^{*}-\mu_{r}}^{n-r} \boldsymbol{E}_{i} W
$$

where $\mu_{r}:=\mu\left(S_{r}\right)$. To this end, let $f \in \mathscr{M}\left(S_{r}\right)$ be such that

$$
f(\beta)=\delta_{\alpha \beta} \quad\left(\beta \in S_{r}\right) .
$$

Observe that

$$
f\left(\boldsymbol{D}_{1}^{*}, \boldsymbol{D}_{2}^{*}, \ldots, \boldsymbol{D}_{s}^{*}\right)-\boldsymbol{E}_{\alpha}^{*} \in \sum_{\beta \notin S_{r}} \mathbb{R} \boldsymbol{E}_{\beta}^{*}
$$

Since

$$
W \subset \sum_{i=r}^{n-r} \boldsymbol{E}_{i}^{*} V^{\otimes n}
$$

by Lemma 2.1(iii), we have

$$
\pi_{W} \boldsymbol{E}_{\beta}^{*} \hat{C}=0 \text { unless } \beta \in S_{r},
$$

from which it follows that

$$
\pi_{W} \boldsymbol{E}_{\alpha}^{*} \hat{C}=\pi_{W} f\left(\boldsymbol{D}_{1}^{*}, \boldsymbol{D}_{2}^{*}, \ldots, \boldsymbol{D}_{s}^{*}\right) \hat{C} .
$$

Let $U$ be the orthogonal complement of the primary $\boldsymbol{T}$-module $\boldsymbol{M} \hat{\boldsymbol{x}}_{0}$ in $V^{\otimes n}$, and let $\pi_{U}: V^{\otimes n} \rightarrow U$ be the orthogonal projection onto $U$. Note that $\pi_{U} \boldsymbol{E}_{0}=\boldsymbol{E}_{0} \pi_{U}=0$ since $\boldsymbol{E}_{0} V^{\otimes n} \subset \boldsymbol{M} \hat{\boldsymbol{x}}_{0}$, so that

$$
\pi_{U} \hat{C} \in \sum_{i=\delta^{*}}^{n} \boldsymbol{E}_{i} V^{\otimes n} .
$$

Moreover, since $\pi_{U}$ is a $\boldsymbol{T}$-homomorphism and since $W \subset U$ by Lemma 4.1, we have

$$
\pi_{W} \boldsymbol{B}^{*} \hat{C}=\pi_{W} \pi_{U} \boldsymbol{B}^{*} \hat{C}=\pi_{W} \boldsymbol{B}^{*} \pi_{U} \hat{C} \quad\left(\boldsymbol{B}^{*} \in \boldsymbol{M}^{*}\right) .
$$

By the definition of $\mu_{r}$ and (32),$f\left(\boldsymbol{D}_{1}^{*}, \boldsymbol{D}_{2}^{*}, \ldots, \boldsymbol{D}_{s}^{*}\right)$ is written as a polynomial in the $\boldsymbol{A}_{\boldsymbol{e}_{i}}^{*}$ with degree at most $\mu_{r}$. For any $\beta, \gamma \in \mathbb{N}^{s}$ with $|\beta|,|\gamma| \leqslant n$, we also have

$$
\boldsymbol{E}_{\beta} \boldsymbol{A}_{\boldsymbol{e}_{i}}^{*} \boldsymbol{E}_{\gamma}=0 \text { if }|| \beta|-| \gamma||>1
$$

by virtue of (4) and (the dual of) (14). Hence it follows from (35), (36), and (37) that

$$
\pi_{W} \boldsymbol{E}_{\alpha}^{*} \hat{C} \in \pi_{W} \sum_{i=\delta^{*}-\mu_{r}}^{n} \boldsymbol{E}_{i} V^{\otimes n}=\sum_{i=\delta^{*}-\mu_{r}}^{n-r} \boldsymbol{E}_{i} W .
$$

This proves (33).

Assume now that $\boldsymbol{E}_{\alpha}^{*} \hat{C}$ is not orthogonal to $W$, i.e., $\pi_{W} \boldsymbol{E}_{\alpha}^{*} \hat{C} \neq 0$. Let

$$
\ell=\min \left\{i: \boldsymbol{E}_{i} \pi_{W} \boldsymbol{E}_{\alpha}^{*} \hat{C} \neq 0\right\} .
$$

By Lemma2.1(iii), $\boldsymbol{E}_{\ell} \pi_{W} \boldsymbol{E}_{\alpha}^{*} \hat{C}$ spans $\boldsymbol{E}_{\ell} W$. In view of Lemma 2.1(ii), (v), we have

$$
\boldsymbol{E}_{r}\left(\boldsymbol{A}_{1}^{*}\right)^{\ell-r} \pi_{W} \boldsymbol{E}_{\alpha}^{*} \hat{C}=\boldsymbol{E}_{r}\left(\boldsymbol{A}_{1}^{*}\right)^{\ell-r} \boldsymbol{E}_{\ell} \pi_{W} \boldsymbol{E}_{\alpha}^{*} \hat{C} \neq 0 .
$$

Since $\pi_{W}$ is a $\boldsymbol{T}_{H}$-homomorphism, it follows from (18) that

$$
0 \neq \boldsymbol{E}_{r} \pi_{W}\left(\boldsymbol{A}_{1}^{*}\right)^{\ell-r} \boldsymbol{E}_{\alpha}^{*} \hat{C}=\left(\theta_{|\alpha|}^{*}\right)^{\ell-r} \boldsymbol{E}_{r} \pi_{W} \boldsymbol{E}_{\alpha}^{*} \hat{C},
$$


i.e., we must have $\ell=r$. However, this contradicts (33) since $\delta^{*}-\mu_{r}>r$. It follows that $\pi_{W} \boldsymbol{E}_{\alpha}^{*} \hat{C}=0$, and the proof is complete.

4.2. Proof of Supplement 3.2. The most important step in the proof of Theorem 3.1 was to establish (33), and the first key observation (35) in this process was based on (34). By Lemma 4.2, (34) can now be improved as follows:

$$
\pi_{W} \boldsymbol{E}_{\beta}^{*} \hat{C}=0 \text { unless } \beta \in S_{r} \backslash K .
$$

Hence it suffices to interpolate on $S_{r} \backslash K$, as desired.

4.3. Proof of Supplement 3.3, At the end of the proof of Theorem 3.1 we used (33) and the assumption $\delta^{*}-\mu_{r}>r$ to show that $\pi_{W} \boldsymbol{E}_{\alpha}^{*} \hat{C}=0$. Observe that we arrive at the same conclusion if we can instead prove that

$$
\pi_{W} \boldsymbol{E}_{\alpha}^{*} \hat{C} \in \sum_{i=r+1}^{n-r} \boldsymbol{E}_{i} W
$$

Let $\delta_{L}^{*}$ denote the scalar in (23), and recall that we are assuming that $\delta_{L}^{*}-\mu_{r}>r$. Then (36) becomes

$$
\pi_{U}\left(\hat{C}-\sum_{\beta \in L} \boldsymbol{E}_{\beta} \hat{C}\right) \in \sum_{i=\delta_{L}^{*}}^{n} \boldsymbol{E}_{i} V^{\otimes n},
$$

from which it follows in the same manner that

$$
\pi_{W} \boldsymbol{F}_{\alpha}^{*}\left(\hat{C}-\sum_{\beta \in L} \boldsymbol{E}_{\beta} \hat{C}\right) \in \sum_{i=\delta_{L}^{*}-\mu_{r}}^{n-r} \boldsymbol{E}_{i} W \subset \sum_{i=r+1}^{n-r} \boldsymbol{E}_{i} W,
$$

where we abbreviate

$$
\boldsymbol{F}_{\alpha}^{*}=f\left(\boldsymbol{D}_{1}^{*}, \boldsymbol{D}_{2}^{*}, \ldots, \boldsymbol{D}_{s}^{*}\right) .
$$

On the other hand, recall that the roles of $\boldsymbol{M}$ and $\boldsymbol{M}^{*}$ are interchanged when we work with the basis $\left\{\hat{\varepsilon}: \varepsilon \in X^{* n}\right\}$ of $V^{\otimes n}$, and observe that $\boldsymbol{E}_{\beta} \hat{C}$ is a scalar multiple of the characteristic vector of $\left(X^{* n}\right)_{\beta} \cap C^{\perp}$ with respect to this basis; cf. (9). Hence, for any $\beta \in L$ and $0 \leqslant i \leqslant t$, it follows from Lemma 4.3 (applied to the dual) that

$$
\begin{aligned}
\boldsymbol{E}_{i} \pi_{W} \boldsymbol{F}_{\alpha}^{*} \boldsymbol{E}_{\beta} \hat{C} & =\boldsymbol{E}_{i} \pi_{W} \pi_{U_{0}} \boldsymbol{F}_{\alpha}^{*} \boldsymbol{E}_{\beta} \hat{C} \\
& =\pi_{W} \boldsymbol{E}_{i} \pi_{U_{0}} \boldsymbol{F}_{\alpha}^{*} \boldsymbol{E}_{\beta} \hat{C} \\
& \in \mathbb{C} \pi_{W} \boldsymbol{A}_{i}^{*} \hat{\iota} \\
& =0,
\end{aligned}
$$

where $\iota=(\iota, \iota, \ldots, \iota)$ is the identity of $X^{* n}$, since $\boldsymbol{A}_{i}^{*} \hat{\boldsymbol{\iota}}=|X|^{n / 2} \boldsymbol{E}_{i} \hat{\mathbf{0}}$ belongs to the primary $\boldsymbol{T}_{H}$-module $\boldsymbol{M}_{H} \hat{\mathbf{0}}$. (Recall that $\boldsymbol{x}_{0}=\mathbf{0}=(0,0, \ldots, 0)$ in this context.) Hence we have

$$
\pi_{W} \boldsymbol{F}_{\alpha}^{*} \boldsymbol{E}_{\beta} \hat{C} \in \sum_{i=t+1}^{n-r} \boldsymbol{E}_{i} W \subset \sum_{i=r+1}^{n-r} \boldsymbol{E}_{i} W \quad(\beta \in L) .
$$

Combining (35), (39), and (40), we obtain (38), and this completes the proof. 


\section{ExAmples}

In this section, we mainly discuss additive codes over various translation association schemes (so that $x_{0}=0$ ).

5.1. Codes with Hamming weight enumerators. Recall that the Hamming weight of $\boldsymbol{x}=\left(x_{1}, x_{2}, \ldots, x_{n}\right) \in X^{n}$ is defined by

$$
\operatorname{wt}(\boldsymbol{x})=\left|\left\{\ell: x_{\ell} \neq 0\right\}\right| \text {. }
$$

The Hamming weight enumerator of an additive code $C$ in $X^{n}$ is then defined by

$$
\operatorname{hwe}_{C}\left(\xi_{0}, \xi_{1}\right)=\sum_{\boldsymbol{x} \in C} \xi_{0}^{n-\mathrm{wt}(\boldsymbol{x})} \xi_{1}^{\mathrm{wt}(\boldsymbol{x})} .
$$

Thus, when working with the Hamming weight enumerator, we are considering codes over the 1-class association scheme $\left(X,\left\{R_{0},(X \times X) \backslash R_{0}\right\}\right)$ with eigenmatrices

$$
P=Q=\left[\begin{array}{cc}
1 & |X|-1 \\
1 & -1
\end{array}\right]
$$

whose extension of length $n$ is the Hamming association scheme $H(n,|X|)$. In particular, we have $\boldsymbol{T}=\boldsymbol{T}_{H}$ in this case. Tanaka [37] showed the following:

Theorem 5.1 ([37, Theorem 5.2, Example 5.5]). Let $C$ be a code in $X^{n}$. Let

$$
\delta=\min \left\{i \neq 0: \boldsymbol{E}_{i}^{*} \hat{C} \neq 0\right\}, \quad \delta^{*}=\min \left\{i \neq 0: \boldsymbol{E}_{i} \hat{C} \neq 0\right\} .
$$

Suppose that an integer $t(1 \leqslant t \leqslant n)$ is such that, for every $1 \leqslant r \leqslant t$, at least one of the following holds:

$$
\begin{gathered}
\left|\left\{i: r \leqslant i \leqslant n-r, \boldsymbol{E}_{i}^{*} \hat{C} \neq 0\right\}\right| \leqslant \delta^{*}-r, \\
\left|\left\{i: r \leqslant i \leqslant n-r, \boldsymbol{E}_{i} \hat{C} \neq 0\right\}\right| \leqslant \delta-r .
\end{gathered}
$$

Then the multiset

$$
\left\{\operatorname{supp}(\boldsymbol{x}): \boldsymbol{x} \in\left(X^{n}\right)_{i} \cap C\right\}
$$

is a t-design for every $0 \leqslant i \leqslant n$.

Observe that Theorem 5.1 strengthens the original Assmus-Mattson theorem (Theorem 1.1). In particular, it does not require that $C$ be linear nor additive. The condition (41) agrees with (21) when $s=1$. Indeed, the proof of Theorem 3.1 reduces to that of Theorem 5.1 for (41). The dual argument shows the result for the case (42). (It seems that the condition dual to (21) does not necessarily lead to the same conclusion as Theorem 3.1 when $s>1$.) On the other hand, Supplements 3.2 and 3.3 refine [37, Remark 7.1], and prove useful as we will see below.

Example 5.2. The Assmus-Mattson-type theorem for additive codes over $\mathbb{F}_{4}$ given by Kim and Pless [23, Theorem 2.7] follows from Theorem 5.1] except their comment on the simplicity of the designs obtained from minimum weight codewords. The additive group of $\mathbb{F}_{4}$ is isomorphic to the Klein four-group $\mathbb{Z}_{2} \times \mathbb{Z}_{2}$, and additive codes over $\mathbb{F}_{4}$ are the same thing as linear Kleinian codes studied by Höhn [20]. It should be noted that giving an (appropriate) inner product on $\mathbb{F}_{4}^{n} \cong\left(\mathbb{Z}_{2} \times \mathbb{Z}_{2}\right)^{n}$, on which concepts like self-orthogonality and self-duality depend, amounts to choosing a group isomorphism $\mathbb{Z}_{2} \times \mathbb{Z}_{2} \rightarrow\left(\mathbb{Z}_{2} \times \mathbb{Z}_{2}\right)^{*}$ satisfying the symmetry (11). This last remark applies to all examples that follow. 
Example 5.3. Let $C$ be an extremal binary Type II code of length $n \equiv 8 \ell(\bmod 24)$ where $\ell \in\{0,1,2\}$. From Theorem 1.1 (or Theorem 5.1) it follows that the words of any fixed weight in $C$ support a $t$-design with $t=5-2 \ell$. Using Bachoc's results on harmonic weight enumerators 2], Bannai, Koike, Shinohara, and Tagami [5, Theorem 6, Remark 5] showed that if one of these (non-trivial) designs is a $(t+1)$ design then so are the others. This observation is also immediate from Supplement 3.2. We note that similar observations hold for extremal Type III codes over $\mathbb{F}_{3}$ and extremal Type IV codes over $\mathbb{F}_{4}$. See also [26].

Example 5.4. Additive codes over $\mathbb{Z}_{4}$ are also referred to as $\mathbb{Z}_{4}$-linear codes. For a $\mathbb{Z}_{4}$-linear code $C$ in $\mathbb{Z}_{4}^{n}$, let

$$
C_{2}=\left(2 \mathbb{Z}_{4}^{n}\right) \cap C
$$

which may also be viewed as a binary linear code (called the torsion code of $C$ ) since $2 \mathbb{Z}_{4} \cong \mathbb{Z}_{2}$. We note that hwe $C_{2}$ is derived immediately from either the complete or the symmetrized weight enumerators of $C$; cf. Subsection 5.2. Shin, Kumar, and Helleseth [30, Theorem 10] proved an Assmus-Mattson-type theorem for $\mathbb{Z}_{4}$-linear codes, and we now claim that Theorem 5.1, together with Supplements 3.2 and 3.3 . always gives at least as good estimate on $t$ as their theorem. First, they assume that $C_{2}$ and $\left(C^{\perp}\right)_{2}$ both satisfy the conclusion of Theorem 5.1. If a (Hamming) weight of $C$ is not a weight of $C \backslash C_{2}$, then the corresponding words of $C$ must all belong to $C_{2}$, and hence by Supplement 3.2 we can exclude that weight from the weights of $C$. The same comment applies to $C^{\perp}$. Second, they assume that the number of non-zero weights of the shortened code of $C^{\perp} \backslash\left(C^{\perp}\right)_{2}$ at some $t$ coordinates is bounded above by $\delta-t$. However, the conclusion of their theorem shows in the end that this number is equal to that of non-zero weights at most $n-t$ in $C^{\perp} \backslash\left(C^{\perp}\right)_{2}$. Hence it follows that this second condition is not weaker than (42).

Remark 5.5. From the Assmus-Mattson-type theorem by Shin et al. mentioned above (or Theorem 5.1) it follows that the words of any fixed weight in the Goethals code or its dual (a Delsarte-Goethals code) over $\mathbb{Z}_{4}$ of length $2^{m}$ with $m$ odd, support a 2-design. However, Shin et al. [30, Corollaries 7 and 8] showed that it is in fact a 3-design, based on what they call an Assmus-Mattson-type approach. See also [24].

5.2. Codes with complete/symmetrized weight enumerators. Let $C$ be an additive code over the ring $\mathbb{Z}_{k}$. Besides hwe ${ }_{C}$, it is also important to consider the complete and the symmetrized weight enumerators defined respectively by

$$
\begin{aligned}
\operatorname{cwe}_{C}\left(\xi_{0}, \xi_{1}, \ldots, \xi_{k-1}\right) & =\sum_{\boldsymbol{x} \in C} \xi_{0}^{n_{0}(\boldsymbol{x})} \xi_{1}^{n_{1}(\boldsymbol{x})} \ldots \xi_{k-1}^{n_{k-1}(\boldsymbol{x})}, \\
\operatorname{swe}_{C}\left(\xi_{0}, \xi_{1}, \ldots, \xi_{e}\right) & =\sum_{\boldsymbol{x} \in C} \xi_{0}^{n_{0}(\boldsymbol{x})} \xi_{1}^{n_{ \pm 1}(\boldsymbol{x})} \ldots \xi_{e}^{n_{ \pm e}(\boldsymbol{x})},
\end{aligned}
$$

where $e=\lfloor k / 2\rfloor$,

$$
\begin{aligned}
n_{i}(\boldsymbol{x}) & =\left|\left\{\ell: x_{\ell}=i\right\}\right| \quad(0 \leqslant i \leqslant k-1), \\
n_{ \pm i}(\boldsymbol{x}) & =n_{i}(\boldsymbol{x})+n_{k-i}(\boldsymbol{x}) \quad(1 \leqslant i \leqslant\lfloor(k-1) / 2\rfloor),
\end{aligned}
$$

and we understand that $n_{ \pm e}(\boldsymbol{x})=n_{e}(\boldsymbol{x})$ if $k$ is even. Thus, for $\mathrm{cwe}_{C}$, the initial association scheme $(X, \mathcal{R})$ is the group association scheme of $\mathbb{Z}_{k}$, which is the 
translation association scheme on $\mathbb{Z}_{k}$ defined by the partition (cf. (5) )

$$
\mathbb{Z}_{k}=\{0\} \sqcup\{1\} \sqcup \cdots \sqcup\{k-1\},
$$

and has eigenmatrices

$$
P=\left[\zeta_{k}^{i j}\right]_{i, j=0}^{k-1}, \quad Q=\left[\zeta_{k}^{-i j}\right]_{i, j=0}^{k-1},
$$

where $\zeta_{k} \in \mathbb{C}$ is a primitive $k^{\text {th }}$ root of unity. For $\operatorname{swe}_{C},(X, \mathcal{R})$ is the association scheme of the ordinary $k$-cycle, which is defined similarly by the partition

$$
\mathbb{Z}_{k}=\{0\} \sqcup\{ \pm 1\} \sqcup \cdots \sqcup\{ \pm e\},
$$

and has eigenmatrices

$$
P=Q=\left[\left(1+\delta_{0,2 j}\right)^{-1}\left(\zeta_{k}^{i j}+\zeta_{k}^{-i j}\right)\right]_{i, j=0}^{e},
$$

where $\delta_{0,2 j}$ is evaluated in $\mathbb{Z}_{k}$. Extensions of the ordinary $k$-cycle are referred to as Lee association schemes [39, 32. We note that

$$
\begin{aligned}
\operatorname{swe}_{C}\left(\xi_{0}, \xi_{1}, \ldots, \xi_{e}\right) & =\operatorname{cwe}_{C}\left(\xi_{0}, \xi_{1}, \xi_{2}, \xi_{3}, \ldots, \xi_{2}, \xi_{1}\right), \\
\operatorname{hwe}_{C}\left(\xi_{0}, \xi_{1}\right) & =\operatorname{swe}_{C}\left(\xi_{0}, \xi_{1}, \ldots, \xi_{1}\right),
\end{aligned}
$$

and that

$$
\operatorname{hwe}_{C_{2}}\left(\xi_{0}, \xi_{1}\right)=\operatorname{swe}_{C}\left(\xi_{0}, 0, \xi_{1}\right) \quad \text { when } k=4 \text {. }
$$

Example 5.6. Our main results are in fact modeled after the Assmus-Mattsontype theorem due to Tanabe [36. Theorem 2] for $\mathbb{Z}_{4}$-linear codes with respect to the symmetrized weight enumerator, so that the latter is a special case of the former. In particular, we can easily find 5-designs from the lifted Golay code over $\mathbb{Z}_{4}$ of length 24 as discussed in 36. See also 6. On the other hand, it is unclear at present whether or not Tanabe's original version of his theorem [34, Theorem 3] is a consequence of our results. It would be an interesting problem to understand 34 , Theorem 3] in terms of the irreducible $\boldsymbol{T}$-modules; cf. [28].

See 19 for a survey on $t$-designs constructed from $\mathbb{Z}_{4}$-linear codes.

Below we discuss the extended quadratic residue codes $X Q_{11}$ of length 12 over small finite fields. That these codes support 3-designs follows from the fact that their automorphism groups contain $\operatorname{PSL}\left(\mathbb{F}_{11}^{2}\right)$ and hence are 3 -homogeneous on the 12 coordinates, but we include these examples in order to demonstrate the use of our results further. Recall again that we only look at the weight enumerators (and linearity) of these self-dual codes. We aim at doing the relevant computations by hand. The first example is a warm-up:

Example 5.7. Consider $C=X Q_{11}$ over $\mathbb{F}_{3}=\mathbb{Z}_{3}$, which is the extended ternary Golay code. We have hwe ${ }_{C}$ and cwe $_{C}$ as follows:

\begin{tabular}{c|cc|c}
\hline wt & $n_{1}$ & $n_{2}$ & \# words \\
\hline \hline 0 & 0 & 0 & 1 \\
\hline 6 & 6 & 0 & 22 \\
\cline { 2 - 4 } & 0 & 6 & 22 \\
\cline { 2 - 4 } & 3 & 3 & 220 \\
\hline 9 & 6 & 3 & 220 \\
\cline { 2 - 4 } & 3 & 6 & 220 \\
\hline \multirow{4}{*}{12} & 12 & 0 & 1 \\
\cline { 2 - 4 } & 0 & 12 & 1 \\
\cline { 2 - 4 } & 6 & 6 & 22 \\
\hline
\end{tabular}


As is well known, the words of fixed (Hamming) weight 6 or 9 support 5-designs by Theorem 1.1. (The one with block size 9 is the non-simple trivial design with constant multiplicity 2.) Set $t=3$. We have $\delta^{*}=6$ and

$$
S_{1}=S_{2}=S_{3}=\{(6,0),(0,6),(3,3),(6,3),(3,6)\} .
$$

Observe that the words with $\left(n_{1}, n_{2}\right)=(6,3)$ and those with $\left(n_{1}, n_{2}\right)=(3,6)$ come in pairs by the correspondence $\boldsymbol{x} \mapsto-\boldsymbol{x}$, so that the words with each of these two complete weight types support the (simple!) trivial design. Hence we may disregard them by Supplement [3.2. i.e., we set $K=\{(6,3),(3,6)\}$. Then $S_{3} \backslash K$ consists of three collinear points in $\mathbb{R}^{2}$, and thus we have $\mu\left(S_{3} \backslash K\right)=2$. Since $2<6-3$, it follows from Theorem 3.1 that the non-trivial 5 -design with block size 6 is partitioned into two 3 -designs (after discarding repeated blocks).

Example 5.8. Consider $C=X Q_{11}$ over $\mathbb{F}_{5}=\mathbb{Z}_{5}$. We have hwe $C$ and swe $C$ as follows:

\begin{tabular}{c|cc|c}
\hline $\mathrm{wt}$ & $n_{ \pm 1}$ & $n_{ \pm 2}$ & \#words \\
\hline \hline 0 & 0 & 0 & 1 \\
\hline 6 & 3 & 3 & 440 \\
\hline 7 & 6 & 1 & 264 \\
\cline { 2 - 4 } & 1 & 6 & 264 \\
\hline 8 & 4 & 4 & 2640 \\
\hline 9 & 7 & 2 & 1320 \\
\cline { 2 - 4 } & 2 & 7 & 1320 \\
\hline 10 & 5 & 5 & 5544 \\
\hline \multirow{2}{*}{11} & 8 & 3 & 1320 \\
\cline { 2 - 4 } & 3 & 8 & 1320 \\
\hline \multirow{2}{*}{12} & 11 & 1 & 24 \\
\cline { 2 - 4 } & 1 & 11 & 24 \\
\cline { 2 - 4 } & 6 & 6 & 1144 \\
\hline
\end{tabular}

We have $\delta^{*}=6$. Observe that Theorem 1.1 nor Theorem 5.1 cannot find designs from the supports of the codewords in this case. On the other hand, set $t=3$, and take $\sigma \in \mathrm{GL}\left(\mathbb{R}^{2}\right)$ such that $\sigma(i, j)=(1 / 5)(2 i+3 j, i-j)$. Then we have

$$
\begin{aligned}
& \sigma\left(S_{1}\right)=\left\{\begin{array}{r}
(6,-1), \\
(5,-1),(5,0),(5,1), \\
(4,-1),(4,0),(4,1), \\
(3,0),(3,1)
\end{array}\right\}, \\
& \sigma\left(S_{2}\right)=\left\{\begin{array}{r}
(5,-1),(5,0), \\
(4,-1),(4,0),(4,1), \\
(3,0),(3,1)
\end{array}\right\}, \\
& \sigma\left(S_{3}\right)=\left\{\begin{array}{r}
(5,-1), \\
(4,-1),(4,0),(4,1), \\
(3,0),(3,1)
\end{array}\right\} .
\end{aligned}
$$

From Supplement 3.4 it follows that $\mu\left(S_{1}\right) \leqslant 4$ and $\mu\left(S_{2}\right) \leqslant 3$. If we apply Supplement 3.4 directly to $\sigma\left(S_{3}\right)$ then we would only obtain $\mu\left(S_{3}\right) \leqslant 3$, but indeed it 
follows that $\mu\left(S_{3}\right)=2$. To see this, let

$$
\begin{aligned}
f_{(5,-1)} & =\left(\xi_{1}-3\right)\left(\xi_{1}-4\right) / 2, \\
f_{(4,-1)} & =-\left(\xi_{1}+\xi_{2}-4\right)\left(\xi_{1}-\xi_{2}-3\right) / 2, \\
f_{(4,1)} & =\left(\xi_{1}+\xi_{2}-3\right)\left(\xi_{1}+\xi_{2}-4\right) / 2, \\
f_{(3,0)} & =\left(\xi_{1}-4\right)\left(\xi_{1}+\xi_{2}-4\right), \\
f_{(3,1)} & =-\left(\xi_{1}-4\right)\left(\xi_{1}+2 \xi_{2}-3\right) / 2, \\
f_{(4,0)} & =1-f_{(5,-1)}-f_{(4,-1)}-f_{(4,1)}-f_{(3,0)}-f_{(3,1)} .
\end{aligned}
$$

Then we have

$$
f_{\alpha}(\beta)=\delta_{\alpha \beta} \quad\left(\alpha, \beta \in \sigma\left(S_{3}\right)\right),
$$

from which it follows that the linear span of the $f_{\alpha}\left(\alpha \in \sigma\left(S_{3}\right)\right)$ is an interpolation space with respect to $\sigma\left(S_{3}\right)$. This shows $\mu\left(S_{3}\right)=2$, as desired. Thus, the condition (21) is satisfied for $r \in\{1,2,3\}$. Theorem 3.1 now shows that the codewords of any fixed symmetrized weight type support 3-designs. This example tells us that looking at swe $_{C}$ may sometimes give a better estimate on $t$ than hwe ${ }_{C}$, even when Supplement 3.2 is not applicable.

Finally, we consider $C=X Q_{11}$ over $\mathbb{F}_{4}=\left\{0,1, \omega, \omega^{2}\right\}$. Note that cwe ${ }_{C}$ makes sense by defining $n_{\omega}(\boldsymbol{x})$ and $n_{\omega^{2}}(\boldsymbol{x})$ in the same manner as above. The eigenmatrices of the group association scheme of $\mathbb{F}_{4}$ are given by

$$
P=Q=\left[\begin{array}{cccc}
1 & 1 & 1 & 1 \\
1 & 1 & -1 & -1 \\
1 & -1 & -1 & 1 \\
1 & -1 & 1 & -1
\end{array}\right]
$$

Example 5.9. Consider $C=X Q_{11}$ over $\mathbb{F}_{4}$. We have hwe $C$ and $\mathrm{cwe}_{C}$ as follows:

\begin{tabular}{c|ccc|c}
\hline wt & $n_{1}$ & $n_{\omega}$ & $n_{\omega^{2}}$ & \#words \\
\hline \hline 0 & 0 & 0 & 0 & 1 \\
\hline 6 & 2 & 2 & 2 & 330 \\
\hline 7 & 5 & 1 & 1 & 132 \\
\cline { 2 - 5 } & 1 & 5 & 1 & 132 \\
\cline { 2 - 5 } & 1 & 1 & 5 & 132 \\
\hline 8 & 4 & 4 & 0 & 165 \\
\cline { 2 - 5 } & 4 & 0 & 4 & 165 \\
\cline { 2 - 5 } & 0 & 4 & 4 & 165 \\
\hline 9 & 3 & 3 & 3 & 1320 \\
\hline 10 & 6 & 2 & 2 & 330 \\
\cline { 2 - 5 } & 2 & 6 & 2 & 330 \\
\cline { 2 - 5 } & 2 & 2 & 6 & 330 \\
\hline 11 & 5 & 5 & 1 & 132 \\
\cline { 2 - 5 } & 5 & 1 & 5 & 132 \\
\cline { 2 - 5 } & 1 & 5 & 5 & 132 \\
\hline 12 & 12 & 0 & 0 & 1 \\
\cline { 2 - 5 } & 0 & 12 & 0 & 1 \\
\cline { 2 - 5 } & 0 & 0 & 12 & 1 \\
\cline { 2 - 5 } & 4 & 4 & 4 & 165 \\
\hline
\end{tabular}


We have $\delta^{*}=6$. Again, Theorem 1.1 cannot find designs from the supports of the codewords. Take $\sigma \in \mathrm{GL}\left(\mathbb{R}^{3}\right)$ such that

$$
\sigma(i, j, k)=(1 / 4)(2 i+j+k, i+2 j+k, i+j+2 k) .
$$

Then we have

$$
\begin{aligned}
& \sigma\left(S_{3}\right)=\left\{\begin{array}{l}
(2,2,2),(2,2,3),(2,3,2),(2,3,3), \\
(3,2,2),(3,2,3),(3,3,2),(3,3,3)
\end{array}\right\}, \\
& \sigma\left(S_{2}\right)=\sigma\left(S_{3}\right) \sqcup\{(4,3,3),(3,4,3),(3,3,4)\}, \\
& \sigma\left(S_{1}\right)=\sigma\left(S_{2}\right) \sqcup\{(4,4,3),(4,3,4),(3,4,4)\} .
\end{aligned}
$$

We claim that $\mu\left(S_{1}\right) \leqslant 4$ and that $\mu\left(S_{2}\right)=\mu\left(S_{3}\right)=3$. First, it is easy to see that $\mu\left(S_{3}\right)=3$ as $\sigma\left(S_{3}\right)$ forms a cube. Next, let

$$
f_{(4,3,3)}=\left(\xi_{1}-2\right)\left(\xi_{1}-3\right) / 2 .
$$

Then we have

$$
f_{(4,3,3)}(\alpha)=\delta_{(4,3,3), \alpha} \quad\left(\alpha \in \sigma\left(S_{2}\right)\right)
$$

We similarly define $f_{(3,4,3)}$ and $f_{(3,3,4)}$. Recall that $\mathscr{M}\left(\sigma\left(S_{3}\right)\right)$ denotes a minimal degree interpolation space with respect to $\sigma\left(S_{3}\right)$. Then it is immediate to see that

$$
\mathscr{M}\left(\sigma\left(S_{3}\right)\right)+\mathbb{R} f_{(4,3,3)}+\mathbb{R} f_{(3,4,3)}+\mathbb{R} f_{(3,3,4)}
$$

is an interpolation space with respect to $\sigma\left(S_{2}\right)$. Since $\mu\left(S_{3}\right) \leqslant \mu\left(S_{2}\right)$, we have $\mu\left(S_{2}\right)=3$. Finally, let for example

$$
f_{(4,4,3)}=\left(\xi_{1}+\xi_{2}-4\right)\left(\xi_{1}+\xi_{2}-5\right)\left(\xi_{1}+\xi_{2}-6\right)\left(\xi_{1}+\xi_{2}-7\right) / 24
$$

so that we have

$$
f_{(4,4,3)}(\alpha)=\delta_{(4,4,3), \alpha} \quad\left(\alpha \in \sigma\left(S_{1}\right)\right)
$$

and a similar argument establishes $\mu\left(S_{1}\right) \leqslant 4$, as desired. Thus, the condition (21) is satisfied for $r \in\{1,2\}$ but fails for $r=3$. Theorem 3.1 now shows that the codewords of any fixed complete weight type support 2-designs. Though this is not the best estimate (i.e., $t=3$ ), Theorem 3.1 still outperforms Theorem 1.1 for this example.

\section{ACKNOWLEDGMENTS}

The authors thank Masaaki Harada for helpful discussions. HT was supported in part by JSPS KAKENHI Grant No. 25400034.

\section{REFERENCES}

[1] E. F. Assmus, Jr. and H. F. Mattson, Jr., New 5-designs, J. Combin. Theory 6 (1969) 122-151.

[2] C. Bachoc, On harmonic weight enumerators of binary codes, Des. Codes Cryptogr. 18 (1999) $11-28$.

[3] E. Bannai, E. Bannai, S. Suda, and H. Tanaka, On relative $t$-designs in polynomial association schemes, Electron. J. Combin. 22 (2015) \#P4.47; arXiv:1303.7163

[4] E. Bannai and T. Ito, Algebraic combinatorics I: Association schemes, Benjamin/Cummings, Menlo Park, CA, 1984.

[5] E. Bannai, M. Koike, M. Shinohara, and M. Tagami, Spherical designs attached to extremal lattices and the modulo $p$ property of Fourier coefficients of extremal modular forms, Mosc. Math. J. 6 (2006) 225-264.

[6] A. Bonnecaze, E. Rains, and P. Solé, 3-colored 5-designs and $\mathbf{Z}_{4}$-codes, J. Statist. Plann. Inference 86 (2000) 349-368.

[7] C. de Boor and A. Ron, On multivariate polynomial interpolation, Constr. Approx. 6 (1990) $287-302$. 
[8] C. de Boor and A. Ron, The least solution for the polynomial interpolation problem, Math. Z. 210 (1992) 347-378.

[9] A. E. Brouwer, A. M. Cohen, and A. Neumaier, Distance-regular graphs, Springer-Verlag, Berlin, 1989.

[10] A. R. Calderbank and P. Delsarte, On error-correcting codes and invariant linear forms, SIAM J. Discrete Math. 6 (1993) 1-23.

[11] A. R. Calderbank, P. Delsarte, and N. J. A. Sloane, A strengthening of the Assmus-Mattson theorem, IEEE Trans. Inform. Theory 37 (1991) 1261-1268.

[12] P. Delsarte, An algebraic approach to the association schemes of coding theory, Philips Res. Rep. Suppl. No. 10 (1973).

[13] P. Delsarte and V. I. Levenshtein, Association schemes and coding theory, IEEE Trans. Inform. Theory 44 (1998) 2477-2504.

[14] M. Gasca and T. Sauer, Polynomial interpolation in several variables, Adv. Comput. Math. 12 (2000) 377-410.

[15] D. Gijswijt, A. Schrijver, and H. Tanaka, New upper bounds for nonbinary codes based on the Terwilliger algebra and semidefinite programming, J. Combin. Theory Ser. A 113 (2006) $1719-1731$.

[16] C. D. Godsil, Generalized Hamming schemes, manuscript (2010); arXiv:1011.1044

[17] T. A. Gulliver and M. Harada, Extremal double circulant type II codes over $\mathbb{Z}_{4}$ and construction of 5-(24, 10, 36) designs, Discrete Math. 194 (1999) 129-137.

[18] M. Harada, New 5-designs constructed from the lifted Golay code over $\mathbb{Z}_{4}$, J. Combin. Des. 6 (1998) 225-229.

[19] T. Helleseth, C. Rong, and K. Yang, On $t$-designs from codes over $\mathbf{Z}_{4}$, Discrete Math. 238 (2001) 67-80.

[20] G. Höhn, Self-dual codes over the Kleinian four group, Math. Ann. 327 (2003) 227-255; arXiv:math/0005266

[21] P. Iliev, A Lie-theoretic interpretation of multivariate hypergeometric polynomials, Compos. Math. 148 (2012) 991-1002; arXiv:1101.1683

[22] P. Iliev and P. Terwilliger, The Rahman polynomials and the Lie algebra $\mathfrak{s l}_{3}(\mathbb{C})$, Trans. Amer. Math. Soc. 364 (2012) 4225-4238; arXiv:1006.5062.

[23] J.-L. Kim and V. Pless, Designs in additive codes over $G F(4)$, Des. Codes Cryptogr. 30 (2003) 187-199.

[24] J. Lahtonen, K. Ranto, and R. Vehkalahti, 3-designs from all $\mathbf{Z}_{4}$-Goethals-like codes with block size 7 and 8, Finite Fields Appl. 13 (2007) 815-827.

[25] W. J. Martin and H. Tanaka, Commutative association schemes, European J. Combin. 30 (2009) 1497-1525; arXiv:0811.2475

[26] T. Miezaki and H. Nakasora, An upper bound of the value of $t$ of the support $t$-designs of extremal binary doubly even self-dual codes, Des. Codes Cryptogr. 79 (2016) 37-46; arXiv: 1311.2122

[27] H. Mizukawa and H. Tanaka, $(n+1, m+1)$-hypergeometric functions associated to character algebras, Proc. Amer. Math. Soc. 132 (2004) 2613-2618.

[28] J. V. S. Morales, On Lee association schemes over $\mathbb{Z}_{4}$ and their Terwilliger algebra, Linear Algebra Appl. 510 (2016) 311-328.

[29] A. Schrijver, New code upper bounds from the Terwilliger algebra and semidefinite programming, IEEE Trans. Inform. Theory 51 (2005) 2859-2866.

[30] D.-J. Shin, P. V. Kumar, and T. Helleseth, An Assmus-Mattson-type approach for identifying 3-designs from linear codes over $\mathbf{Z}_{4}$, Des. Codes Cryptogr. 31 (2004) 75-92.

[31] J. Simonis, MacWilliams identities and coordinate partitions, Linear Algebra Appl. 216 (1995) 81-91.

[32] P. Solé, The Lee association scheme, in: G. Cohen and P. Godlewski (eds.), Coding theory and applications, Lecture Notes in Computer Science, vol. 311, Springer-Verlag, Berlin, 1988, pp. $45-55$.

[33] J. N. Srivastava and D. V. Chopra, Balanced arrays and orthogonal arrays, in: J. N. Srivastava (ed.), A survey of combinatorial theory, North-Holland, Amsterdam, 1973, pp. 411-428.

[34] K. Tanabe, An Assmus-Mattson theorem for $\mathbf{Z}_{4}$-codes, IEEE Trans. Inform. Theory 46 (2000) $48-53$.

[35] K. Tanabe, A new proof of the Assmus-Mattson theorem for non-binary codes, Des. Codes Cryptogr. 22 (2001) 149-155. 
[36] K. Tanabe, A criterion for designs in $\mathbb{Z}_{4}$-codes on the symmetrized weight enumerator, Des. Codes Cryptogr. 30 (2003) 169-185.

[37] H. Tanaka, New proofs of the Assmus-Mattson theorem based on the Terwilliger algebra, European J. Combin. 30 (2009) 736-746; arXiv:math/0612740

[38] H. Tanaka, R. Tanaka, and Y. Watanabe, The Terwilliger algebra of a $Q$-polynomial distanceregular graph with respect to a set of vertices, in preparation.

[39] H. Tarnanen, On extensions of association schemes, in: H. Laakso and A. Salomaa (eds.), The very knowledge of coding, University of Turku, Institute for Applied Mathematics, Turku, 1987, pp. $128-142$.

[40] P. Terwilliger, The subconstituent algebra of an association scheme I, J. Algebraic Combin. 1 (1992) 363-388.

[41] P. Terwilliger, The subconstituent algebra of an association scheme II, J. Algebraic Combin. 2 (1993) 73-103.

[42] P. Terwilliger, The subconstituent algebra of an association scheme III, J. Algebraic Combin. 2 (1993) 177-210.

[43] P. Terwilliger, The displacement and split decompositions for a $Q$-polynomial distance-regular graph, Graphs Combin. 21 (2005) 263-276; arXiv:math.CO/0306142

[44] P. Terwilliger, Six lectures on distance-regular graphs, lecture notes, De La Salle University, 2010; http://www.math.wisc.edu/ terwilli/teaching.html.

Graduate School of Information Sciences, Tohoku University, Sendai, Japan

E-mail address: moralesjohnvince@ims.is.tohoku.ac.jp

Research Center for Pure and Applied Mathematics, Graduate School of Information Sciences, Tohoku University, Sendai, Japan

E-mail address: htanaka@tohoku.ac.jp 